\title{
Protective effect of high n-3/n-6 PUFA ratio on acute hypoxic-ischemic brain damage in mfat-1 transgenic mice and possible inflammation-related targets identified by transcriptome analysis
}

\section{xue geng}

Nanjing Medical University

Meng Wang

Nanjing Medical University

Yunjun Leng

Nanjing Medical University

Lin Li

Nanjing Medical University

Haiyuan Yang

Nanjing Medical University

Yifan Dai

Nanjing Medical University

Ying Wang ( $\sim$ ywang@njmu.edu.cn )

Nanjing Medical University

\section{Research}

Keywords: Hypoxia-ischemic brain damage, mfat-1 transgenic mice, RNA-seq, Neuroinflammation, GPR120 receptor

Posted Date: October 20th, 2020

DOI: https://doi.org/10.21203/rs.3.rs-93477/v1

License: (c) (1) This work is licensed under a Creative Commons Attribution 4.0 International License. Read Full License 


\section{Abstract}

\section{Background}

Acute hypoxic-ischemic brain damage (HIBD) occurs not only in newborns but also in adults. It is associated with series of cellular and biochemical pathways that lead to neuronal injury. N-3 polyunsaturated fatty acids (PUFAs) have been reported to improve neuron functions via G proteincoupled receptor 120 signal pathway in cells or with exogenous supplementation. Possible protective targets and underlying mechanisms of high proportion of $n-3 / n-6$ PUFAs contained in the brains of mfat1 transgenic mice on HIBD-induced adult brain damage needed to be further investigated.

\section{Methods}

The adult C57BL/6J (WT) and mfat-1 transgenic mice adopted HIBD model. A gas chromatograph was used to determine the composition of PUFAs. Neurological deficit scores test, TTC staining and Nissl staining were employed to evaluate the neuroprotective effects. Cleaved-caspase 3 and TUNEL experiment were used to detect apoptosis after injury. Inflammatory factors were detected by ELISA assay. RNA-sequencing analysis was processed and the differential expressed genes were verified by real-time quantitative PCR. Key factors related to inflammation were detected by immunofluorescence and western blot.

\section{Results}

The mfat- 1 transgenic mice with high ratio of $n-3 / n-6$ PUFAs in brain tissues were showed to have protective effects on HIBD-induced brain damage by reduced infarct range and greatly improved neurobehavioral defects. Further analysis revealed that the level of neuronal necrosis, apoptosis and inflammation induced by brain injury were relatively low. RNA-seq analysis showed multiple pathways and targets involved in this process. Significant activation of GPR120, reduction of phosphorylation of TAK1 and NF-KB P65 in the downstream of the pro-inflammatory pathway were found in the brains of mfat-1 mice on HIBD.

\section{Conclusions}

The study showed that mfat- 1 transgenic mice had protective effects on HIBD-induced brain injury by multiple pathways. Activation of GPR120 and reduction of related pro-inflammatory pathway involved in this process, which may improve or prevent dangerous perioperative and postoperative complications, innovate clinical intervention strategy and potentially benefit more patients.

\section{Background}

Cerebral hypoxic-ischemic damage mostly occurs in the perinatal period of newborns [1, 2], but acute hypoxic-ischemic injury in adults due to cardiac arrest caused by perioperative period and asphyxia should also not be underestimated [3, 4]. Perioperative acute cerebral ischemic stroke is the main risk 
factor for stroke, encephalopathy and cognitive decline after cardiothoracic surgery, and which may lead to poor prognosis and increased mortality [5-7]. Preoperative prophylaxis against perioperative stroke is an appealing concept. In previous studies, several pharmacological and non-pharmacological methods of neuroprotection have been studied in experimental studies and animal models [8, 9]. Among which, dietary n-3 PUFAs proved to have multiple beneficial effects, such as improved cognitive performance, anti-inflammatory effects, reduced cardiovascular disease risk and increased neuroplasticity [10-13]. PUFAs are a class of fatty acids enriched in brain which is necessary for organisms to maintain a physiologically stable environment and function [14-16]. Traditionally, they can be divided into two categories: n-3 PUFAs and n-6 PUFAs. The major n-3 PUFAs mainly include short-chain alpha-linolenic acid (ALA,18:3n-3), and the longer-chain eicosapentaenoic acid (EPA, 20:5n-3), docosapentaenoic acid (DPA, 22:5n-3) and docosahexaenoic acid (DHA, 22:6n-3). The major dietary n-6 PUFAs is linoleic acid (LA, 18:2n-6) and together with ALA are the PUFAs consumed in largest amounts $[17,18]$. Contrary to higher intake of n-3 PUFAs, epidemiological observations and intervention studies had shown that lower n-3 PUFAs intake increases the risk of many neurological disorders including ischemic stroke due to increasing western diets by multi targets and pathways $[19,20]$. G protein-coupled receptors (GPCRs) are members of the largest family of cell membrane protein receptors, which are targets of many drugs and regulate lots of physiological processes. GPR120 was one of the orphan receptors that could be stimulated by n-3 PUFAs [21, 22]. Evidences showed that activation of GPR120 by n-3 PUFAs exerts antiinflammatory effects in vast kinds of cells, such as hypothalamic neurons, macrophages and mature adipocytes $[23,24]$.

In order to study the function of endogenous high proportion of n-3 PUFAs on acute hypoxic-ischemic brain injury, mfat- 1 transgenic mice were used in current study. The fat- 1 gene encoded an n-3 fatty acid desaturase that could introduce a double bond into n- 6 fatty acids at the n-3 position of their hydrocarbon chains to form n-3 fatty acids, hence converting n-6 PUFAs to n-3 PUFAs [25]. In mfat- 1 transgenic mice, the coding region of $C$. elegans fat- 1 cDNA was optimized to enhance the expression of fat- 1 in mammalian cells [26]. Therefore, mfat- 1 transgenic mice were ideal for addressing the effects of $n-3 / n-6$ ratio in the body tissues for elimination of the need to manipulate the diet, which will avoid the possible confounding effects of dietary supplements in the study [27, 28]. So far, mfat-1 transgenic mice have been showed to play kinds of roles in various diseases, such as preventing cardiovascular disease and myocardial infarction, reducing inflammation, participating in immune regulation of the body and associating with cognitive performance among the old [29-31]. But to date, its function on acute hypoxicischemic brain injury and underlying mechanism still not clearly addressed. In this research, HIBD model was employed to successfully simulate the possible perioperative acute hypoxia-ischemic stroke in adult mice [32].

\section{Methods}

\section{Animal models and treatments}


The mfat- 1 transgenic mice were obtained by prokaryotic microinjection as previous report [26], the coding region of $\mathrm{C}$. elegans fat- $1 \mathrm{cDNA}$ was optimized to enhance the expression of fat- 1 in mammalian cells. The mfat-1 heterozygotes of C57BL/6J and wild-type (WT) C57BL/6J mice were crossed to produce mfat- 1 transgene mice and WT littermates. The genotypes of the mice were identified by polymerase chain reaction (PCR) amplification. Experimental mice were bred in Animal Core Facility of Nanjing Medical University. Feeding conditions: temperature: $22 \pm 2^{\circ} \mathrm{C}$; relative humidity: $50-70 \%$; 12 -hour cycle lighting, free intake of food and water. All animal experiments were approved by the Institutional Animal Care and Use Committee (IACUC) of Nanjing Medical University, with protocol number of IACUC-1911024. Every effort was made to minimize animal suffering.

\section{Acute hypoxic-ischemic brain damage (HIBD) model}

The establishment of a perioperative acute hypoxic-ischemic stroke model is based on the Vannucci mode [32]. The offspring mice obtained by mating of mfat-1 transgenic mice and wild-type mice weighing $22 \pm 2 \mathrm{~g}$ were selected for modeling grouping. After isoflurane ether anesthesia ( $2 \%)$, the left common carotid artery was exposed and ligated with 6-0 nylon wire, the above operations were performed under a stereo microscope (Nikon, Tokyo, Japan). After suture, hypoxia was performed in the hypoxia chamber (Tianchen XL-2CL, Nanjing, China) for 50 minutes. $92 \%$ nitrogen and $8 \%$ oxygen were pumped into the hypoxia chamber. Body temperature was maintained at $37.0 \pm 0.5^{\circ} \mathrm{C}$ throughout surgery. The sham group underwent the same anesthesia and exposure as the surgery group, ignoring ligation and hypoxia. This model has a mortality rate of about $10 \%$.

\section{Gas chromatography analysis of fatty acid profiles}

Fatty acid composition of brain tissue was determined by capillary gas chromatography (Agilent 7890A, CA, USA). Briefly, the total lipids were extracted by the Fatty Acid Extraction kit (Sigma-Aldrich, Missouri, USA), and then dry under nitrogen for transesterification immediately. Fatty acids were methylated with boron trifluoride and methanol (heat at $70^{\circ} \mathrm{C}$ for 1 hour). Add $1 \mathrm{~mL}$ of hexane and $1 \mathrm{~mL}$ of distilled water and then vortex and centrifuge at $500 \mathrm{x} g$ for 5 minutes. Reconstitute the trans esterified lipids with hexane and transfer to a GC vial. Methylated fatty acids were then analyzed via gas chromatography flame ionization detection on gas chromatograph as previously described [33]. Peaks were identified by comparison with authenticated standards (Sigma-Aldrich, Missouri, USA). Fatty acid components were identified by comparison of retention times with those of authentic standards. The ratio of $n-3 / n-6$ was calculated by the areas of peaks. Data are expressed as molar percent of fatty acids.

\section{Longa score}

Behavior deficit was taken by Longa's [34] 5-point scale at 24h after HIBD. A score of 0: no neurological deficit; a score of 1: failure to extend left forepaw fully; a score of 2: circling to the left; a score of 3: falling to the left; and a score of 4: did not walk spontaneously and had a depressed level of consciousness. Mice without symptoms of neurological impairment or dying after HIBD were rejected, and other rats were recruited. 


\section{Berderson score}

Mice were held gently by the tail, suspended one meter above the floor, and observed for forelimb flexion. The evaluation standards of Bederson method were divided into 4 grades, normal grade 0 : no observable deficit; moderate grade 1: forelimb flexion; severe grade 2: decreased resistance to lateral push (and forelimb flexion) without circling; grade 3: same behavior as grade 2, with circling [35].

\section{Rotarod test}

For the rotating test, an accelerating rotarod (Giliang DigBehv-010, Shanghai, China) was used as described previously [36, 37], which accelerated in speed of $20 \mathrm{rpm}$ over a $5 \mathrm{~min}$ period. Record the time for the mouse to fall for the first time and the number of drops (mice were reloaded on rotarod as soon as possible after falling) within 300 s. mice were acclimatized to the rotarod for three trials, with an intertrial interval of $30 \mathrm{~min}$.

\section{2,3,5-Triphenyte-trazoliumchloride (TTC) staining}

After anesthesia, fresh brain tissue was taken immediately, washed with pre-cooled buffer solution ( $1 \mathrm{X}$ PBS) and directly put into quick-frozen at $-20{ }^{\circ} \mathrm{C}$ for 20-30 minutes. The brain was cut into $2-3 \mathrm{~mm}$ tissue blocks with a lycra blade in the brain tank. Then put it into the pre-preheated TTC incubation solution (Sigma-Aldrich, Missouri, USA), placed in the oven at $37^{\circ} \mathrm{C}$ and incubated in the dark for $15 \mathrm{~min}$. The reaction between TTC reagent and dehydrogenase in normal tissues is red, while the dehydrogenase activity in ischemic tissues is reduced and cannot be reacted, thus presenting a pale color. The Image Pro Plus software was used to analysis the infarct volume. Infarct volume $(\%)=($ right ischemic pale area / left brain area + right brain area) $\times 100$.

\section{Nissl staining and hematoxylin and eosin staining}

After perfusion and fixation, drying, samples were dewaxed with xylene and hydrated with alcohol of class gradient concentration. Coronal brain sections (4um) were used to observe the change of the gross morphology by Hematoxylin-Eosin staining and Nissl staining, including brain hemisphere swelling, subcortical petechial hemorrhage, tissue necrosis, nerve cell loss, and inflammatory cell infiltration. The damaged neurons appear vacuolated and pale blue. Normal cells have relatively large, plump nidellae with a darker bluish tint.

\section{Immunohistochemical analysis}

Immunofluorescence staining was carried out to detect GPR120 expression in mice after HIBD. Paraffinembedded formaldehyde fixed specimens were cut into 4um thick slices, dewaxed with xylene, and rehydrated with a series of graded alcohols. Microwave high temperature antigen repair in citric acid buffer ( $3 \%$ trisodium citrate and $0.3 \%$ sodium citrate). Brain serial coronal sections were washed with PBS before fixed with $4 \%$ paraformaldehyde at room temperature for $30 \mathrm{~min}$. Subsequently, they were incubated with a blocking solution ( $5 \% \mathrm{FBS})$ for $30 \mathrm{~min}$ at $37^{\circ} \mathrm{C}$. Then, they were incubated with anti- 
GPR120 antibody (Santa Cruz Biotechnology, CA, USA) overnight at $4{ }^{\circ} \mathrm{C}$. The antibodies used in this section also illustrated in supplementary Table S1. On the following day, they were washed with PBS and incubated with secondary antibodies anti-IgG Conjugates (Invitrogen, Carlsbad, CA, USA) for 1h and 4',6diamidino-2-phenylindole (DAPI) for $1 \mathrm{~min}$ at room temperature in the dark. Images were obtained using a confocal microscope (CarlZeiss LSM710, Oberkochen, German).

\section{Terminal deoxynucleotidyl transferase dUTP nick-end labeling (TUNEL) assay}

TUNEL staining was performed according to the manufacturer's instructions (KeyGEN BioTECH, Nanjing, China) for quantification of neuronal cell death [38].Paraffin sections $(4 \mu \mathrm{m})$ were dried for $1 \mathrm{~h}$ at $70^{\circ} \mathrm{C}$, and deparaffinized in xylene and graded ethanol solutions. Images were obtained using a confocal microscope (CarlZeiss LSM710, Oberkochen, German). The TUNEL-positive cells were counted in the cortex, hippocampus and striatum in three separate fields for each animal by an observer who was blind to the experimental condition. Data was expressed as ratio of TUNEL-positive neurons (\%).

\section{Enzyme-linked immunosorbent assay (ELISA)}

The concentrations of IL- $1 \beta, \mathrm{IL}-6$, and TNF- $\alpha$ in ischemic penumbra in brain of each group were quantified using an ELISA kit (Elabscience Biotechnology, Wuhan, China) according to the manufacturer's instruction. Absorbance at $450 \mathrm{~nm}$ was recorded and the concentration of the target protein was calculated according to the standard curve and normalized against the protein of the samples. Result was expressed as $\mathrm{pg} / \mathrm{mg}$ protein.

\section{RNA-seq analysis}

Total RNA extraction

Total RNA was extracted from the tissues using Trizol (Invitrogen, Carlsbad, CA, USA) according to manual instruction. Ischemic side brain tissues were ground into powder by liquid nitrogen, followed by being homogenized and rested horizontally. The mix was centrifuged, then the supernatant was transferred into a new EP tube with chloroform/isoamyl alcohol (24:1). The mix was shacked vigorously for $15 \mathrm{~s}$, and then centrifuged, the upper aqueous phase where RNA remained was transferred into a new tube with equal volume of supernatant of isopropyl alcohol, then centrifuged at $4^{\circ} \mathrm{C}$. After deserting the supernatant, the RNA pellet was washed twice with $75 \%$ ethanol, then the mix was centrifuged at $4^{\circ} \mathrm{C}$ to collect residual ethanol, followed by the pellet air dry in the biosafety cabinet. Finally, DEPC-treated water was added to dissolve the RNA. Subsequently, total RNA was qualified and quantified using a Nano Drop and Agilent 2100 bioanalyzer (Thermo Fisher Scientific, MA, USA).

mRNA Library Construction

Oligo(dT)-attached magnetic beads were used to purified mRNA. Purified mRNA was fragmented into small pieces with fragment buffer at appropriate temperature. Then First-strand cDNA was generated using random hexamer-primed reverse transcription, followed by a second-strand cDNA synthesis. 
afterwards, A-Tailing Mix and RNA Index Adapters were added by incubating to end repair. The cDNA fragments obtained from previous step were amplified by PCR, and products were purified by Ampure XP Beads, then dissolved in EB solution. The product was validated on the Agilent Technologies 2100 bioanalyzer for quality control. The double stranded PCR products from previous step were heated denatured and circularized by the splint oligo sequence to get the final library. The single strand circle DNA (ssCir DNA) was formatted as the final library. The final library was amplified with phi29 to make DNA nanoball (DNB) which had more than 300 copies of one molecular, DNBs were loaded into the patterned nanoarray and single end 50 bases reads were generated on BGIseq500 platform (BGIShenzhen, China).

Bioinformatics analysis

Primary sequencing data produced by RNA-Seq (raw reads) were subjected to quality control (QC). The sequencing data was filtered with SOAPnuke (v1.5.2) by (1) Removing reads containing sequencing adapter; (2) Removing reads whose low-quality base ratio (base quality less than or equal to 5 ) is more than $20 \%$; (3) Removing reads whose unknown base (' $N$ ' base) ratio is more than $5 \%$, afterwards clean reads were obtained and stored in FASTQ format. The clean reads were mapped to the reference genome using HISAT2 (v2.0.4). Bowtie2 (v2.2.5) was applied to align the clean reads to the reference coding gene set, then expression level of gene was calculated by RSEM (v1.2.12). The heatmap was drawn by pheatmap (v1.0.8) according to the gene expression in different samples. Essentially, differential expression analysis was performed using the DESeq2(v1.4.5) with $Q$ value $\leq 0.05$. To take insight to the change of phenotype, GO (http://www.geneontology.org/) and KEGG (https://www.kegg.jp/) enrichment analysis of annotated different expressed gene was performed by Phyper (https://en.wikipedia.org/wiki/Hypergeometric_distribution) based on Hypergeometric test. The significant levels of terms and pathways were corrected by $Q$ value with a rigorous threshold ( $Q$ value $\leq$ $0.05)$ by Bonferroni.

\section{Western blotting}

Western blotting was performed according to the manufacturer's specification. The ischemic side brain tissue samples were collected at 24h after HIBD. Proteins were extracted by homogenizing in RIPA buffer (Sigma-Aldrich, Missouri, USA) with phenylmethanesulfonyl fluoride (PMSF) and phosphatase inhibitor (Bimake, TX, USA) and further centrifuged at $12,000 \mathrm{rpm}$ at $4{ }^{\circ} \mathrm{C}$ for $10 \mathrm{~min}$. The concentrations were determined with BCA protein assay kit (Thermo Fisher Scientific, MA, USA). the samples were separated by $8-12 \%$ SDS-PAGE gel (Bio-Rad, CA, USA) and then transferred to polyvinylidene fluoride (PVDF) (BioRad, CA, USA) membranes. Subsequently, the membranes were blocked in 5\% BSA (Sigma-Aldrich, Missouri, USA) for $1 \mathrm{~h}$ at room temperature following incubation with primary antibodies overnight at 4 ${ }^{\circ} \mathrm{C}$. Dilutions for primary and secondary antibodies were listed in supplementary Table S1. Membranes were washed three times in TBST and specific binding was visualized by ECL reaction (Bio-Rad, CA, USA). The density of bands was detected using an imaging densitometer, and the gray value of the bands was quantified using ImageJ Software (version 1.41). 


\section{Real-time PCR}

Total RNA was extracted from the ischemic cerebral cortices at $24 \mathrm{~h}$ after HIBD using Trizol reagent (Invitrogen, CA, USA) according to the manufacturer's protocol. The quantity of total RNA was measured with a UV spectrophotometer (Thermo Fisher Scientific, MA, USA). Next, reverse transcription was performed using a cDNA synthesis kit HiScript II Q RT SuperMix (Vazyme Biotech, China). Quantitative PCR was performed with ChamQ SYBR qPCR Master Mix (Vazyme Biotech, China) at the following conditions (denaturing at $95^{\circ} \mathrm{C}$ for $30 \mathrm{~s}$, followed by 40 cycles of $95^{\circ} \mathrm{C}$ for $10 \mathrm{~s}$ and $60^{\circ} \mathrm{C}$ for $30 \mathrm{~s}$ ) and detected by StepOnePlus Real-Time PCR Systems (Applied Biosystems; MA, USA). The expression of target genes was measured in triplicate and normalized to $\beta$-actin as an internal control. The $-\Delta \Delta C t$ values of each group were analyzed, and mRNA expression levels were normalized to $2^{-\Delta \Delta C t}$. Primers are listed in Supplementary table S2.

\section{Statistical analysis}

GraphPad Prism 8.0.1 software (GraphPad Software Inc, La Jolla, CA) was used to analyze data and form the graphs in this work (including which tests were performed, exact $P$ values, and sample sizes). Simply, one-way ANOVA with a test for linear trend, Tukey test was used as appropriate to analyze parametric statistics. The statistics of neural score longa score and Berderson score data use rank sum test in nonparametric test. At least three independent experiments were applied to collect effective data. Bias was avoided by making sure that assessor was blinded to collecting and analyzing data. $P<0.05$ was considered significant. Average values represent the mean $\pm S D$. *: $P<0.05$; $* *: P<0.01$; $* \star *: P<0.001$.

\section{Results}

\section{Expression of the mfat- 1 transgene elevated the ratio of $n-3 / n-6$ PUFAs}

The genotypes of the mice were identified by PCR and showed in Supplementary Fig.S1. The whole brain tissues of mfat- 1 transgenic and WT mice were analyzed for fatty acid composition using GC. As showed in Fig. $1 \mathrm{~A}$, the ratio of $n-3 / n-6$ PUFAs was significantly higher than that in WT mice in the same litter $(86.89 \pm 9.61$ in mfat-1 vs. $62.11 \pm 3.38$ in WT, $p<0.05)$ due to decreased proportion of $n-6$ PUFAs, including AA and LA, increased expression of n-3 PUFAs, including EPA, DPA, ALA and DHA. Moreover, we performed RNA-seq analysis on the whole brains of WT and mfat-1 mice in the same litters on physiological conditions. A total of 46 differentially expressed genes (DEGs) was identified in supplementary table S3, including 43 up-regulated genes and 3 down-regulated genes. Fig. 1B showed the cluster heat map of all DEGs. GO and KEGG pathway analysis were used for functional annotation to determine the biological significance of the differential clustering of all DEGs mRNA and related enrichment pathways in Supplementary Fig. S2. Most of these significant pathways were closely related to the synthesis and metabolism of unsaturated fatty acids, neurogenesis and neuron development, and signal transduction. The above results showed that the brains of mfat- 1 transgenic mice not only contain a high proportion of $n-3 / n-6$ PUFAs, but also differs from WT mice in terms of gene expression levels 
related to polyunsaturated fatty acid metabolism and neurotrophic metabolism. This was in line with the expectation of using mfat- 1 transgenic mice to study neuroprotection in this study. In sum, mfat- 1 mice are ideal for addressing the effects of $n-3 / n-6$ ratio in the brain tissues.

\section{Neuroprotective effects of high ratio of n-3/n-6 PUFAs in mfat-1 mice on HIBD}

In order to investigate the protective effect of high ratio of n-3/n-6 PUFAs in mfat- 1 transgenic mice on brain lesions induced by HIBD, brain tissue was carefully checked. In Fig. 2A, compared with mfat-1 transgenic mice brain, WT brain tissue showed that the central part of the ischemia is more swollen and more bleeding around. In addition, H/E staining found in Fig. 2B, in WT+HIBD group, small focal hemorrhage occurred in the cerebral cortex, and a large number of red blood cells overflowed around the damaged vessel wall, however, this type of hemorrhage rarely appeared in the mfat- $1+$ HIBD group (pointed by the arrow). Infarction is an important manifestation of brain injury, and its area can directly reflect the degree of injury [39]. In Fig. 2C, TTC staining exhibited that the infarct volume in the mfat-1 group was significantly reduced (39.32 \pm 6.34 in WT+HIBD vs. $15.42 \pm 6.06$ in WT+HIBD, $p<0.05)$. In Fig. 2D, $\mathrm{H} /$ E staining showed in the WT+HIBD group, much more hippocampus and cortical neurons were lost and more typical red neurons were observed which defined by severely chromatin agglutination, shrank and deformed cell body, dark red cytoplasm, unclear outline and appearance of foam cells [40] (pointed by the arrow). While in mfat-1+HIBD groups, there was only slight condensation of chromatin in the hippocampus and cortical neurons, but no significant neuron loss and typical red neurons were observed. These results indicated that mfat- 1 mice containing high ratio of endogenous $n-3 / n-6$ PUFAs in mfat- 1 transgenic mice have a neuroprotective effect on HIBD.

\section{High ratio of n-3/n-6 PUFAs in mfat-1 mice on HIBD improved neurobehavioral performance}

Classical Longa score and Berderson score were used as indicators to evaluate the neurological defect and neurobehavioral performance at 24h after HIBD. As showed in Fig.3A, all mice in sham groups had a neurologic grade of 0 , while in the HIBD group, mfat-1 mice obtained a lower Longa score compared with WT group and exhibited better neurobehavior. In addition, Berderson score in Fig. 3B showed similar results. Subsequently, we investigated motor coordination and balance using the rotarod test at $24 \mathrm{~h}$ after HIBD. In the sham operation groups, the mice had coordinated limbs on the rotating rod and had a strong balance ability, although they would rotate with the rotating rod due to fatigue in the later stage. In HIBD groups, WT mice got more significant motor function defects than mfat-1 mice exhibited by shorter latency to fall and increased times of drops within 300s compared with mfat- 1 mice (Fig. 3C, 3D). These results reflected that mfat- 1 mice possessed low neurological deficits and relatively better motor coordination and balance maintenance ability compared with WT mice at $24 \mathrm{~h}$ after HIBD.

\section{Endogenous high ratio of n-3/n-6 PUFAs in mfat-1 mice protected neurons against HIBD-induced neuronal apoptosis}

Nissl staining was used to identify apoptosis and loss of neurons in the damaged area. Typical neuronal lesions in the hippocampus and cortex in the WT+HIBD group were showed in Fig. 4A: the central NissI 
corpuscle was dissolved, mainly manifested as neuron swelling and rounding; nucleolar got larger, the cytoplasmic central Nissl body disintegrated, dissolved and the disappeared. Next, western blotting was performed to verify the expression level of apoptotic relative protein (Fig. 4B). $24 \mathrm{~h}$ after HIBD, cleaved caspase-3 showed higher expression in the ischemic hemisphere of WT group compared with that in mfat-1 group. In Fig. 4C, TUNEL staining further confirmed that apoptotic neurons in mfat-1 group were significantly reduce compared with WT group in the hippocampus as well as the cortex and striatum. The above results demonstrated that mfat- 1 transgenic mice could prevent apoptosis damage caused by acute hypoxia-ischemia in the perioperative period.

\section{The mfat-1 transgenic mice showed reduced inflammation on HIBD}

Mounting evidence suggests that inflammation is a key contributor to the severity of CNS hypoxiaischemia injury [41, 42] After HIBD, their ipsilateral hemispheres may display an inflammatory response, we evaluated changes in the mRNA and protein levels of pro-inflammatory cytokines at $24 \mathrm{~h}$ after HIBD insult by q-PCR and ELISA. HIBD caused a significant increasement in the secretion of IL-1 $\beta$, IL-6, and TNF- $a$ compared to sham treatment as expected. However, mRNA expression level of IL-6 $(137.03 \pm 11.33$ in WT+HIBD vs. $56.94 \pm 10.76$ in mfat-1+HIBD, $p<0.01)$, IL-1 $13(86.82 \pm 2.06$ in WT+HIBD vs. $14.77 \pm 11.97$ in mfat- $1+$ HIBD, $p<0.01)$ and TNF-a $(20.27 \pm 0.66$ in WT+HIBD vs. $5.58 \pm 3.52$ in mfat- $1+H I B D, p<0.01)$ significantly decreased in mfat-1 transgenic mice compared with WT+HIBD group (Fig. 5A-C). Correspondingly, a significant decrease of protein levels of IL-1 $\beta(92.37 \pm 0.85$ in WT+HIBD vs. $77.23 \pm 1.39$ in mfat-1+HIBD, p <0.001), IL-6 (206.25 \pm 8.62 in WT+HIBD vs. $144.21 \pm 30.39$ in mfat- $1+H I B D, p<0.01)$, and TNF-a $(218.34 \pm 5.25$ in WT+HIBD vs. $171.51 \pm 8.60$ in mfat $1+H I B D, p<0.001)$ had also been detected (Fig. $5 D-F)$. To sum, these results illustrated that high proportion of $n-3 / n-6$ PUFAs contained in the brains of mfat-1 transgenic mice was a potent suppressor of HIBD-induced inflammation.

\section{RNA-seq transcriptome analysis of DEGs on HIBD}

After acute hypoxia-ischemia, a series of pathological processes are triggered in the lesion area. RNA-seq transcriptome was used to identify the full gene expression profile of WT and mfat-1 mice in the same litters at 24 hours after HIBD with ipsilateral hemispheres. After DEGs screening, 1936 down-regulated genes and 1315 up-regulated genes were obtained showing by volcano map (Fig. 6A). Next, we performed KEGG pathway analysis on differentially down-regulated genes and up-regulated genes respectively. TOP20 enrichment pathways in the down-regulated and up-regulated genes were showed in the form of bubble chart (Fig. 6B1 and 6C1). And then, we used Cytoscape software to perform enrichment analysis on the DEGs involved in the significant pathways (Fig. 6B2 and 6C2). Statistical analysis found that in the down-regulated 1,936 DEGs, 247 enrichment pathways were involved, mainly involving cytokine-cytokine receptor interaction, osteoclast differentiation, ribosome, TNF signaling pathway, NOD-like receptor signaling pathway, PI3K-Akt signaling pathway, MAPK signaling pathway, chemokine signaling pathway, neuroactive ligand-receptor interaction and apoptosis, etc. Most of the down-regulated pathways were closely related to inflammatory response, indicating that mfat-1 transgenic mice could inhibit the inflammatory response induced by HIBD. In contrast, among the 1,315 
up-regulated DEGs, there were 247 enriched pathways involved, mainly involving phosphatidylinositol signaling system, glutamatergic synapse, aldosterone synthesis and secretion, neurotrophin signaling pathway, phospholipase D signaling pathway, axon guidance, long-term depression and calcium signaling pathway, etc. Compared with WT mice, most of these up-regulated pathways were closely related to nerve growth and differentiation, cholinergic synapse development, and nervous system signal transduction. Neurotrophic factors and growth factors could promote tissue repair and vascular remodeling [43]. They were critical for the recovery of ischemic brain. This detailed analysis showed for the first time that the genetic regulatory network of neurotrophic factors, its receptors and the protein kinases that influence metabolism might mediate brain damage induced by ischemia and hypoxia.

\section{Verification of the DEGs on HIBD}

In the enrichment network analysis, we found that IL-6, IL-1ß, TNF-a, AP-1, Ifnar2, Mmp3, T|r4, Cxc11, $\mathrm{Cxcl} 2, \mathrm{Cxcl} 3, \mathrm{Ccl} 2$ and $\mathrm{Ccl} 12$ etc. were involved in the regulation of most of significant pathways in all down-regulated genes, and these genes all belong to inflammatory signaling pathway. The mRNA and protein expression levels of pro-inflammatory factors IL-6, IL1 $\beta$, and TNF- $a$ have been verified in Fig. 5. which were consistent with RNA-Seq results. In addition, we also found that Mapk7, Pik3ca, Pik3r3, Itpr3, Foxo3, Itrp1, Mapk12, Grid2 and Map2k7 are involved in the regulation of most of significant pathways in all up-regulated genes, and these genes all belonged to neurotrophin signaling pathway. Then, String public database was used to perform interaction analysis on these selected genes (Fig.7A and 7C). Next, qPCR was used to verify the expression of these screened differential genes, and the qPCR results were consistent with RNA-seq transcriptome analysis (Fig.7B, 7D).

\section{Activation of GPR120, suppression phosphorylation of TAK1 and NF-KB involved in protection against HIBD in mfat-1 mice by alleviating inflammation}

As an important receptor for n-3 PUFAs, the expression of GPR120 in the mouse brain is mainly concentrated in the olfactory bulb, cerebral cortex, and a small amount of expression in the hippocampus (Allen brain map data). And recent study showed that the expression of GPR120 was remarkably increased in the microglia, neurons, astrocytes of penumbra of cortex after ischemic injury [44]. Indeed, in our case, the expression level of GPR120 was found higher in mfat-1 mice compared with WT mice with or without HIBD, and its expression was verified not only from RNA-seq but also by immunofluorescence analysis and western blot (Fig. 7A ,7B, 7C). These findings illustrated the existence and participation of GPR120 in HIBD itself and its further protective effects in mfat-1 mice. Previous research reported that through GPR120 signaling pathway, n-3 PUFAs pretreatment could inhibit the pro-inflammatory responses in downstream such as the phosphorylation level of TAK1 and NF-KB $[45,46]$. Therefore, we performed western blot on p-NF-KB P65 and p-TAK1. As expected, the phosphorylation level of NF-KB P65 and TAK1 protein in the brains significantly decreased in the mfat-1 mice compared to the WT mice on HIBD (Fig. 7D and 7E). Mechanistically, we concluded that mfat- 1 transgenic mice alleviated inflammation by activating the GPR120 receptor, reducing the phosphorylation of TAK1 and NF-KB P65 and then significantly inhibited the release of pro-inflammatory cytokines: IL-1 $\beta$, TNF-a and IL-6. 


\section{Discussion}

One of the significant risk factors that patients undergoing major cardiothoracic surgery is ischemic stroke [47]. In order to prevent subsequent occurrence of central nervous system diseases, related events must be either reduced in severity or prevented completely. Different methods had been tried including the use of pharmacological drugs and other non-pharmacological methods [48-50]. Previous studies have demonstrated that exogenous and endogenous n-3 PUFAs exerted protective effects in ischemic stroke after focal cerebral ischemia $[16,20]$. However, what is protective effect of endogenous high proportion of $\mathrm{n}-3 / \mathrm{n}-6$ PUFAs on HIBD model and the underlying mechanism involved in this process still need to be thoroughly researched.

Well-controlled mfat- 1 transgenic mice on a normal diet were used in current study, and the ratio of $n-3 / n$ 6 PUFAs (DHA+EPA+DPA+ALA/LA+AA) was statistically higher in the brain tissues of littermates in mfat1 mice. Without interference from long-term dietary of other bioactive compounds, the transgenic mice proved to be a better model compared with exogenous supplementation. Consistent with our pervious report [26], the n-3 PUFAs in the brains of mfat-1 mice not only contain a higher proportion of DHA, but also contain EPA and DPA that are almost absent in the brain of WT mice, which leading to an increase in the overall $n-3 / n-6$ PUFA ratio. The overall increase in n-3 PUFAs should be better than exogenous supplementation of specific kind of n-3 PUFAs. Whole-transcriptome deep sequencing of the whole brain tissues of mfat- 1 transgenic mice were performed on normal condition and on HIBD for the first time, although transcriptome sequencing of the hippocampus of mfat-1 mice had been reported [51]. First, the transcription level of mfat- 1 transgene was proved to be at a stable and high expression level in the brain tissues. And then, we found that many DEGs, such as SCD1, GPX3, DUSP1, Susd2, Ngfr, were involved in the synthesis, metabolism and transportation of fatty acids in the brain [52-54]. This further suggested that mfat- 1 transgenic mice were suitable model to research protective effect of endogenous high n- $3 / n-6$ PUFA ratio in the brain.

In this study, mfat-1 transgenic mice had protective effects on HIBD-induced brain damage by significantly reduced infarct range, greatly improved neurobehavioral defects, relatively lower level of neuronal necrosis, apoptosis and inflammation. Although many studies have revealed its pathological mechanism [55,56], the molecular details of the subsequent events after HIBD and the underlying protective mechanism from mfat-1 mice still needed to be further elucidated. RNA-seq technology was used to identify the full gene expression profiles of WT and mfat- 1 mice at $24 \mathrm{~h}$ after acute ischemia and hypoxia. To our knowledge, this is so far the first study to profile the gene expression changes and key pathways on HIBD in mfat-1 mice. The results provided a multi-faceted research directions and targets for the subsequent study of mfat- 1 transgenic mice in ischemic and hypoxic diseases. Among them, antiinflammatory signaling pathway was the most significant one. Inflammation was one of the crucial factors of secondary neuronal injury after global $\mathrm{HI}$ neonatal perinatal period $[57,58]$, however, the inflammatory related targets and pathways after HIBD in adults during perioperative period are still unclear. As an important receptor for n-3 PUFAs, GPR120 was found mainly expressed in the olfactory bulb, cerebral cortex and hippocampus on physiological condition (Allen brain atlas), and activated 
expression in this HIBD model. GPR120 activation has been shown to produce anti-inflammatory effects in previous studies $[44,59]$. GPR120 couples with b-arrestin2 to induce receptor endocytosis, which in turn inactivates phosphorylation of NF-KB, thereby providing a mechanism to inhibit inflammation signaling pathways [44]. Therefore, we speculated that the activation of GPR120 might protect brain injury in HIBD model by alleviating the inflammation in the ischemic area. As expected, we found that GPR120 expression in the penumbra of the affected side of WT and mfat- 1 mice was significantly increased after acute hypoxia-ischemic injury. It was worth noting that in the sham operation group, the expression of GPR120 in mouse brain tissue of WT mice and mfat-1was different as well. Although the expression level of GPR120 in the penumbra of WT mice was significantly activated within 24 hours after acute ischemia and hypoxia, but the downstream TAK1 activated by the receptor after endocytosis was not weakened, neuronal necrosis and apoptosis caused by inflammation have not improved as a result. On the contrary, the expression of GPR120 in mfat-1 mice was at a high level under physiological conditions. $24 \mathrm{~h}$ after acute ischemic hypoxia, GPR120 is overactivated further, which significantly inhibits phosphorylation of TAK1 and NF-KB induced by acute ischemic stroke. Therefore, the TAK1-NFKB inflammatory pathway in the brain is significantly interfered. Of note, endogenous n-3 PUFAs might also exert anti-inflammatory effects through other mechanisms. For example, recent studies have shown that metabolites of n-3 PUFAs such as resolvins, protectins and maresin may play a role in improving inflammation [60]. Although the CT imaging of adult and neonatal individual brains was different for hypoxia- ischemia damage, the mechanisms of inflammation and apoptosis induced by hypoxia- ischemia injury were similar.

\section{Conclusions}

In summary, we concluded the endogenous high proportion of n-3/n-6 PUFAs in the brains of mfat- 1 mice had a certain neuroprotective trend for acute hypoxia-ischemic stroke that occurs during the perioperative period. Among the multiple complicated pathological protection mechanisms, current improved neurological outcomes mainly from activation of GPR120 pathway in alleviation of inflammation. Understanding of these insights can serve as the basis for broadening the scope of treatment of hypoxiaischemia-related encephalopathy during the perioperative period and finally benefit more patients.

\section{Abbreviations}

ALA: Alpha-linolenic acid; AP-1: Jun proto-oncogene; BCA: Bicinchonininc acid; Ccl2: Chemokine (C-C motif) ligand 1; CCl12: Chemokine (C-C motif) ligand 12; CNS: Central nervous system; Cxcl1: Chemokine (C-X-C motif) ligand 1; Cxcl2: Chemokine (C-X-C motif) ligand 2; Cxcl3: Chemokine (C-X-C motif) ligand 3; DAPI: 4',6-diamidino-2-phenylindole; DEGs: Differentially expressed genes; DEPC: Diethyl pyrocarbonate; DHA: Docosahexaenoic acid; DPA: Docosapentaenoic acid; ECL: Enhanced chemiluminescent; ELISA: Enzyme-linked immunosorbent assay; EPA: Eicosapentaenoic acid; FBS: Fetal bovine serum; Foxo3: Forkhead box 03; G0: Gene Ontology; GPR120: G-protein coupled receptor 120; GPX3: Glutathione peroxidase 3; HIBD: Hypoxic ischemic brain damage; Ifnar2: Interferon alpha and beta receptor subunit 2; 
IL-13: Interleukin 1 beta; IL-6: Interleukin 6; Itrp1: Inositol 1,4,5-trisphosphate receptor 1; Itrp3: Inositol 1,4,5trisphosphate receptor 3; KEGG: Kyoto Encyclopedia of Genes and Genomes; LA: linoleic acid; Map2k7: Mitogen-activated protein kinase kinase 7; Mapk12ロMitogen-activated protein kinase 12; Mmp3: Matrix metallopeptidase 3; NF-KB: nuclear factor of kappa light polypeptide gene enhancer in B cells 1 ; Ngfr: Nerve growth factor receptor; Ntrk3: Neurotrophic tyrosine kinase, receptor, type 3; PBS: Phosphatebuffered saline; PCR: Polymerase chain reaction; Pik3ca: Phosphatidylinositol-4,5-bisphosphate 3-kinase catalytic subunit alpha; Pik3r3: Phosphoinositide-3-kinase regulatory subunit 3; PMSF:

Phenylmethanesulfonyl fluoride; $\mathbf{p}-\mathbf{N F}$ KB: Phosphorylation- nuclear factor of kappa light polypeptide gene enhancer in B cells 1; p-TAK1: Phosphorylation- Mitogen-activated protein kinase kinase kinase 7; PUFAs: Polyunsaturated fatty acid; PVDF: Polyvinylidene fluoride; qPCR: Real-time quantitative polymerase chain reaction; RIPA: Radio-Immunoprecipitation Assay; SCD1: Stearoyl- Coenzyme A desaturase 1; SDS-PAGE: Sodium dodecyl sulfate polyacrylamide gel electrophoresis; Susd2: Sushi domain containing 2; TAK1: Mitogen-activated protein kinase kinase kinase 7; TBST: Tris-Buffered Saline and Tween 20; TIr4: Toll-like receptor 4; TNF-a: Tumour necrosis factor alpha-like; TTC: 2,3,5-Triphenyte-trazoliumchloride; TUNEL: Terminal deoxynucleotidyl transferase dUTP nick-end labeling; WT: Wild-type

\section{Declarations}

\section{Acknowledgements}

Not applicable.

\section{Funding}

This work was supported by grants from the National Natural Science Foundation of China (no. 31701283 \& 81970164), the National Key R\&D Program of China (no. 2017YFC1103701 \& 2017YFC1103702), the Jiangsu Key Laboratory of Xenotransplantation (BM2012116).

\section{Availability of data and materials}

All the datasets and materials supporting the conclusions of this article are presented in the manuscript.

\section{Authors' contributions}

$X G$ and $Y W$ contributed to the design and writing of the manuscript. $X G, M W$, and $Y L$ did several experiments and helped in the acquisition of data. XG, YW and HY did analysis and interpretation of data. HY helped in the statistical analysis and revision of the manuscript. LL helped in the technical support and purchasing of reagents. YD, YW and HY helped in the technical support, obtaining of funding. All authors read and approved the final manuscript.

\section{Ethics approval}


Mice were housed according to international standard conditions and all animal experiments conformed to the guidelines for the care and use of laboratory animals by the National Institutes of Health $(\mathrm{NIH})$ and approved by the Institutional Animal Care and Use Committee (IACUC) of Nanjing Medical University (Nanjing, China).

Competing interests

The authors declare that they have no competing interests.

\section{References}

1. Hou K, Li G, Zhao J, Xu B, Xu K: Correction to: Bone mesenchymal stem cell-derived exosomal microRNA-29b-3p prevents hypoxic-ischemic injury in rat brain by activating the PTEN-mediated Akt signaling pathway. Journal of Neuroinflammation 2020, 17(1).

2. Jiao M, Li X, Chen L, Wang X, Yin H: Neuroprotective effect of astrocyte-derived IL-33 in neonatal hypoxic-ischemic brain injury. Journal of Neuroinflammation 2020.

3. Gutierrez LG, Rovira à, Portela LAP, Leite CDC, Lucato LT: CT and MR in non-neonatal hypoxicischemic encephalopathy: radiological findings with pathophysiological correlations. Neuroradiology 2010, 52(11):949-976.

4. Wagner, Franca, Haenggi, Matthias M, Grunt, Sebastian, Weck, Anja, Z'Graggen, Werner J: The value of susceptibility-weighted imaging (SWI) in patients with non-neonatal hypoxic-ischemic encephalopathy. Resuscitation 2015.

5. Grau AJ, Eicke M, Burmeister C, Hardt R, Schmitt E, Dienlin S: Risk of Ischemic Stroke and Transient Ischemic Attack Is Increased up to 90 Days after Non-Carotid and Non-Cardiac Surgery. Cerebrovascular Diseases 2017.

6. Selim M: Perioperative stroke. N Engl J Med 2007, 356(7):706-713.

7. Yu S, Li P: Cognitive declines after perioperative covert stroke: Recent advances and perspectives. Current Opinion in Anesthesiology 2020, 33.

8. González-Nieto D, Fernández-Serra R, Pérez-Rigueiro J, Panetsos F, Martinez-Murillo R, Guinea GV: Biomaterials to Neuroprotect the Stroke Brain: A Large Opportunity for Narrow Time Windows. Cells 2020, 9(5).

9. Yang, Liu, Yanying, Yin, Qiao-Li, Lu, Ying, Dan, Mei-Song, Xu: Vinpocetine in the treatment of poststroke cognitive dysfunction: A protocol for systematic review and meta-analysis. Medicine 2019.

10. Simopoulos AP: Evolutionary aspects of diet: the omega-6/omega-3 ratio and the brain. Molecular Neurobiology 2011, 44(2):203-215.

11. Fang G, Shi B, Wu K, Chen S, Gao X, Xiao S, Kang JX, Li W, Huang R: The Protective Role of Endogenous n-3 Polyunsaturated Fatty Acids in Tau Alzheimer's Disease Mouse Model. International Journal of Neuroscience 2018, 129:1-24. 
12. Schunck W-H, Konkel A, Fischer R, Weylandt K-H: Therapeutic potential of omega-3 fatty acid-derived epoxyeicosanoids in cardiovascular and inflammatory diseases. Pharmacology \& Therapeutics 2017:S0163725817302668.

13. Yehuda, S.: Polyunsaturated fatty acids as putative cognitive enhancers. Medical Hypotheses 2012, 79(4):456-461.

14. Librowski T, Patkowska-Soko $\AA_{i}^{\prime} \# 130$ Be, Herbinger G, Bodkowski R, Czy $\AA^{1} \frac{1}{4}$ K, Stanis $\AA_{i} \# 130$, Lochy $\AA_{i}^{\prime} \# 132$ a, ski, PÄł \#153 J, kala et al: Omega-3 Fatty Acids and their Role in Central Nervous System - A Review. Current Medicinal Chemistry 2016, 23(8):--

15. Saini RK, Keum YS: Omega-3 and omega- 6 polyunsaturated fatty acids: Dietary sources, metabolism, and significance - A review. Life ences 2018, 203:255-267.

16. Bazinet RP, Laye S: Polyunsaturated fatty acids and their metabolites in brain function and disease. Nat Rev Neurosci 2014, 15(12):771-785.

17. Baker EJ, Miles EA, Burdge GC, Yaqoob P, Calder PC: Metabolism and functional effects of plantderived omega-3 fatty acids in humans. Progress in lipid research 2016, 64:30-56.

18. Wiktorowska-Owczarek A, Berezińska M, Nowak JZ: PUFAs: Structures, Metabolism and Functions. Advances in Clinical and Experimental Medicine 2015, 24(6):931-941.

19. Simopoulos AP: Evolutionary aspects of diet: the omega-6/omega-3 ratio and the brain. $\mathrm{Mol}$ Neurobio/ 2011, 44(2):203-215.

20. Hu X, Zhang F, K. Leak R, Zhang W, Iwai M, A. Stetler R, Dai Y, Zhao A, Gao Y, Chen J: Transgenic Overproduction of Omega-3 Polyunsaturated Fatty Acids Provides Neuroprotection and Enhances Endogenous Neurogenesis After Stroke. Current Molecular Medicine 2013, 13(9):--

21. Karakua-Juchnowicz H, Róg J, Juchnowicz D, Morylowska-Topolska J: GPR120: Mechanism of action, role and potential for medical applications. Postepy Hig Med Dosw 2017, 71:942-953.

22. Oh DY, Walenta E, Akiyama TE, Lagakos WS, Lackey D, Pessentheiner AR, Sasik R, Hah N, Chi TJ, Cox $\mathrm{JM}$ et al: A Gpr120-selective agonist improves insulin resistance and chronic inflammation in obese mice. Nat Med 2014, 20(8):942-947.

23. Dragano NRV, Solon C, Ramalho AF, De Moura RF, Razolli DS, Christiansen E, Azevedo C, Ulven T, Velloso LA: Polyunsaturated fatty acid receptors, GPR40 and GPR120, are expressed in the hypothalamus and control energy homeostasis and inflammation. Journal of Neuroinflammation 2017, 14(1):91.

24. Hasan AU, Ohmori K, Konishi K, Igarashi J, Hashimoto T, Kamitori K, Yamaguchi F, Tsukamoto I, Uyama T, Ishihara $Y$ et al: Eicosapentaenoic acid upregulates VEGF-A through both GPR120 and PPARgamma mediated pathways in 3T3-L1 adipocytes. Mol Cell Endocrinol 2015, 406:10-18.

25. Bilal S, Haworth O, Wu L, Weylandt KH, Levy BD, Kang JX: Fat-1 transgenic mice with elevated omega-3 fatty acids are protected from allergic airway responses. Biochimica Et Biophysica Acta 2011, 1812(9):1164-1169.

26. Yu J, Yang H, Fang B, Zhang Z, Wang Y, Dai Y: mfat-1 transgene protects cultured adult neural stem cells against cobalt chloride-mediated hypoxic injury by activating Nrf2/ARE pathways. J Neurosci 
Res 2018, 96(1):87-102.

27. Kang JX: Fat-1 Transgenic Mice: A New Model for Omega-3 Research. Prostaglandins Leukot Essent Fatty Acids 2007, 77(5-6):263-267.

28. Kang J: Fat-1 transgenic mice convert 1-6 to n-3 fatty acids. Nature 2004, 427.

29. Guo XF, Gao J, Li J, Li D: fat-1 mice prevent high-fat plus high-sugar diet-induced non-alcoholic fatty liver disease. Food \& Function 2017:10.1039.C1037F001050H.

30. Huan, Ma, Peipei, Chen, Chuanlan, Sang, Daozheng, Huang, Qingshan, Geng: Modulation of apoptosis-related microRNAs following myocardial infarction in fat-1 transgenic mice vs wild-type mice. Journal of Cellular \& Molecular Medicine 2018.

31. Rahman MM, Halade GV, Bhattacharya A, Fernandes G: The fat-1 transgene in mice increases antioxidant potential, reduces pro-inflammatory cytokine levels, and enhances PPARY and SIRT-1 expression on a calorie restricted diet. Oxidative Medicine \& Cellular Longevity 2009, 2(5):307.

32. Vannucci RC, Vannucci SJ: A model of perinatal hypoxic-ischemic brain damage. Journal of Neuroscience Research 2010, 835(Frontiers of Neurology A Symposium in Honor of Fred Plum):234249.

33. Bibus D, Lands B: Balancing proportions of competing omega-3 and omega- 6 highly unsaturated fatty acids (HUFA) in tissue lipids. Prostaglandins Leukotrienes \& Essential Fatty Acids 2015, 99:1923.

34. Longa EZ, Weinstein PR, Carlson S, Cummins R: Reversible middle cerebral artery occlusion without craniectomy in rats. Stroke; a journal of cerebral circulation 1989, 20(1):84.

35. Bederson JB, Pitts LH, Tsuj M, Nishimura MC, Bartkowski H: Rat middle cerebral artery occlusion: Evaluation of the model and development of a neurologic examination. Stroke 1986, 17(3):276-472.

36. Enam SF, Kader SR, Bodkin N, Lyon JG, Bellamkonda RV: Evaluation of M2-like macrophage enrichment after diffuse traumatic brain injury through transient interleukin-4 expression from engineered mesenchymal stromal cells. Journal of Neuroinflammation 2020, 17(1).

37. Stroobants S, Gantois I, Pooters T, D’Hooge R: Increased gait variability in mice with small cerebellar cortex lesions and normal rotarod performance. Behavioural Brain Research 2013, 241(Complete):32-37.

38. Wang Z, Zhou F, Dou Y, Tian X, Liu C, Li H, Shen H, Chen G: Melatonin Alleviates Intracerebral Hemorrhage-Induced Secondary Brain Injury in Rats via Suppressing Apoptosis, Inflammation, Oxidative Stress, DNA Damage, and Mitochondria Injury. Translational Stroke Research 2018.

39. Devin, W., McBride, Damon, Klebe, Jiping, Tang, John, H., Zhang: Correcting for Brain Swelling's Effects on Infarct Volume Calculation After Middle Cerebral Artery Occlusion in Rats. Translational Stroke Research 2015.

40. Yuan Q, Li R, Yang H, Zhang G: Effects of Reperfusion on Neuronal Changes and Macrophagic Response after Transient Focal Ischemia- Reperfusion of Brain in Rats. JOURNAL OF WEST CHINA UNIVERSITY OF MEDICAL ENCES 1999, 30(2):155-157, 137. 
41. Anna-Maj, Albertsson, Dan, Bi, Luqi, Duan, Xiaoli, Zhang, Jianmei: The immune response after hypoxia-ischemia in a mouse model of preterm brain injury. Journal of Neuroinflammation 2014.

42. Vexler ZS, Yenari MA: Does inflammation after stroke affect the developing brain differently than adult brain? Developmental Neuroscience 2009, 31(5):378-393.

43. Wang YC, Sanchez E, Sardari M, Carvalho TSD, Hermann DM: Postacute Delivery of GABAA a5 Antagonist Promotes Postischemic Neurological Recovery and Peri-infarct Brain Remodeling. Stroke 2018, 49(10).

44. Ren Z, Chen L, Wang Y, Wei X, Zeng S, Zheng Y, Gao C, Liu H: Activation of the Omega-3 Fatty Acid Receptor GPR120 Protects against Focal Cerebral Ischemic Injury by Preventing Inflammation and Apoptosis in Mice. The Journal of Immunology 2018.

45. Wei TT, Yang LT, Guo F, Tao SB, Cheng L, Huang RS, Ma L, Fu P: Activation of GPR120 in podocytes ameliorates kidney fibrosis and inflammation in diabetic nephropathy. Acta Pharmacol Sin 2020.

46. Yin J, Li H, Meng C, Chen D, Chen Z, Wang Y, Wang Z, Chen G: Inhibitory effects of omega-3 fatty acids on early brain injury after subarachnoid hemorrhage in rats: Possible involvement of $G$ proteincoupled receptor $120 / \beta$-arrestin2/TGF- $\beta$ activated kinase-1 binding protein-1 signaling pathway. Int $J$ Biochem Cell Biol 2016, 75:11-22.

47. Costa MA, Gauer MF, Gomes RZ, Schafranski MD: Risk factors for perioperative ischemic stroke in cardiac surgery. Rev Bras Cir Cardiovasc 2015, 30(3):365-372.

48. Bhutta AT, Schmitz ML, Swearingen C, James LP, Wardbegnoche WL, Lindquist DM, Glasier CM, Tuzcu V, Prodhan P, Dyamenahalli U: Ketamine as a neuroprotective and anti-inflammatory agent in children undergoing surgery on cardiopulmonary bypass: A pilot randomized, double-blind, placebocontrolled trial. Pediatr Crit Care Med 2012, 13(3):328-337.

49. Lai TW, Zhang S, Wang YT: Excitotoxicity and stroke: Identifying novel targets for neuroprotection. Progress in Neurobiology 2014, 115:157-188.

50. Simon, J., Mitchell, and, Alan, F., Merry, and, Christopher, Frampton: Cerebral Protection by Lidocaine During Cardiac Operations: A Follow-Up Study. The Annals of Thoracic Surgery 2009.

51. Ménesi D, Kitajka K, Molnár E, Kis Z, Belleger J, Narce M, Kang JX, Puskás LG, Das UN: Gene and protein expression profiling of the fat-1 mouse brain. Prostaglandins Leukotrienes \& Essential Fatty Acids 2009, 80(1):33-42.

52. Dobrzyn A, Dobrzyn P, Lee SH, Miyazaki M, Ntambi JM: Stearoyl-CoA desaturase-1 deficiency reduces ceramide synthesis by downregulating serine palmitoyltransferase and increasing $\beta$-oxidation in skeletal muscle. Am J Physiol Endocrinol Metab 2005, 288(3):E599.

53. Jin Q, Li R, Hu N, Xin T, Zhu P, Hu S, Ma S, Zhu H, Ren J, Zhou H: DUSP1 alleviates cardiac ischemia/reperfusion injury by suppressing the Mff-required mitochondrial fission and Bnip3-related mitophagy via the JNK pathways. Redox biology 2018, 14:576.

54. Nadjar Y, Triller A, Bessereau JL, Dumoulin A: The Susd2 protein regulates neurite growth and excitatory synaptic density in hippocampal cultures. Molecular and Cellular Neuroence 2015, 65:8291. 
55. Hirayama Y, Koizumi S: Hypoxia-independent mechanisms of HIF-1a expression in astrocytes after ischemic preconditioning. Glia 2017, 65(3):523-530.

56. Vetrovoy O, Sarieva K, Lomert E, Nimiritsky P, Rybnikova E: Pharmacological HIF1 Inhibition Eliminates Downregulation of the Pentose Phosphate Pathway and Prevents Neuronal Apoptosis in Rat Hippocampus Caused by Severe Hypoxia. Journal of Molecular Neuroence 2019, 70(5).

57. Bhalala US, Koehler RC, Kannan S: Neuroinflammation and Neuroimmune Dysregulation after Acute Hypoxic-Ischemic Injury of Developing Brain. Frontiers in Pediatrics 2015, 2:144.

58. Harukuni I, Bhardwaj A: Mechanisms of brain injury after global cerebral ischemia. Neurologic Clinics 2006, 24(1):1-21.

59. Im, Dong-Soon: FFA4 (GPR120) as a fatty acid sensor involved in appetite control, insulin sensitivity and inflammation regulation. Molecular Aspects of Medicine 2017:S009829971730064X.

60. Serhan CN: Pro-resolving lipid mediators are leads for resolution physiology. Nature 2014, 510(7503):92-101.

\section{Supplemental Information}

Additional file 1:Table S1.

The antibodies used in this study.

Additional file 2: Table S2.

The primers used in qPCR in this study.

Additional file 3: Figure S1.

Mouse Genotyping. The adult $(22 \pm 2 \mathrm{~g})$ mfat- 1 transgenic mice and WT littermates were identified by genotyping using polymerase chain reaction (PCR) amplification. Genomic DNA was extracted from mice tails using a TIANamp Genomic DNA Kit (TianGen, Beijing, China). The PCR primers used for the mfat-1 gene were forward 5'-GGACCTTGGTGAAGAGCATCCG-3' and reverse 5'-GCGTCGCAGAAGCCAAAC-3'. PCR product was $438 \mathrm{bp}$. The PCR conditions were as follows: $95^{\circ} \mathrm{C}$ for $5 \mathrm{~min}(1 \mathrm{cycle}), 95^{\circ} \mathrm{C}$ for $30 \mathrm{~s}, 65^{\circ} \mathrm{C}$ for $30 \mathrm{~s}$, and $72^{\circ} \mathrm{C}$ for $60 \mathrm{~s}$ (35cycles). The PCR products were separated by $1.5 \%$ agarose gel electrophoresis.

\section{Additional file 4: Table S3.}

RNA-seq analysis of all differential genes and their expression annotations in WT and mfat-1 transgenic mice in the same litter (ARC=Average Read Count).

Additional file 5: Figure S2. 
KEGG pathway enrichment result between mfat-1 and WT mice. According to the KEGG pathway annotation classification, use the phyper function in the $\mathrm{R}$ software to perform enrichment analysis, calculate the Pvalue, and then perform FDR correction on the Pvalue.

\section{Figures}

A

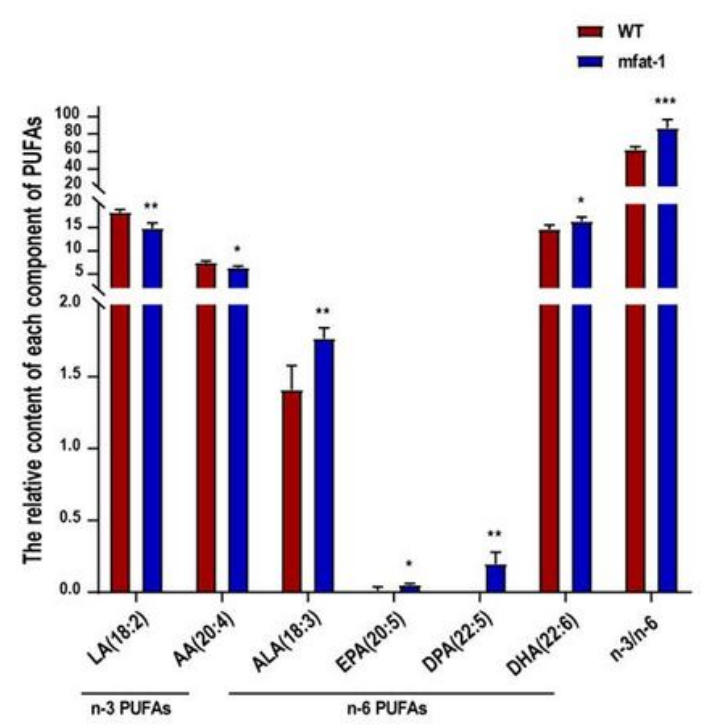

B

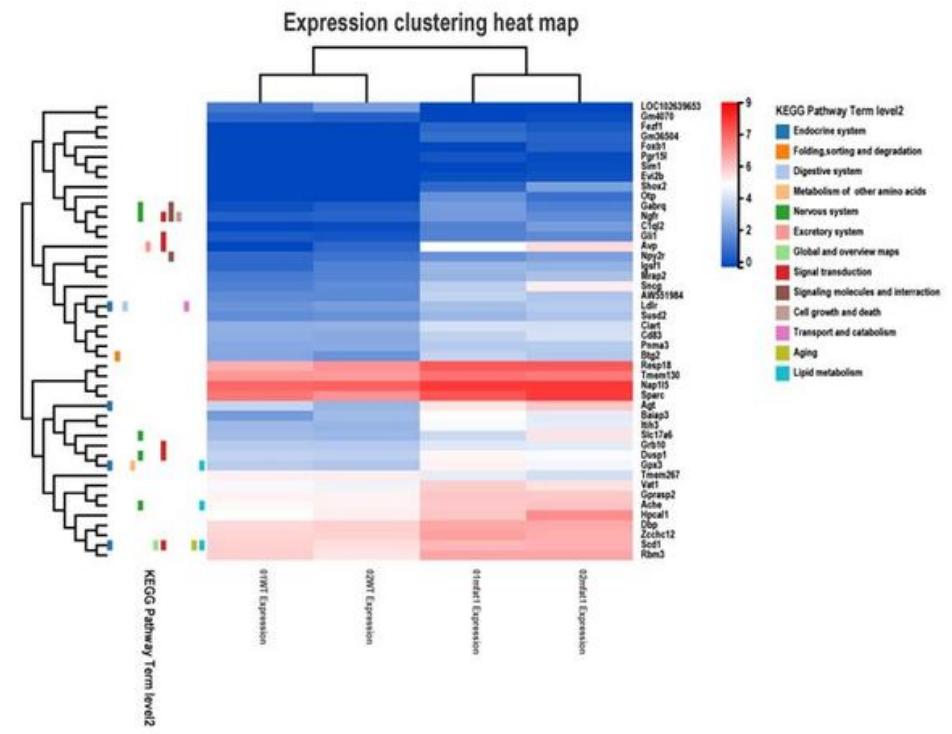

\section{Figure 1}

Expression of the mfat- 1 transgene elevated the ratio of n-3/n-6 PUFAs. A Brain tissues were collected from mfat- 1 transgenic mice and WT control littermates. Compositions of n-3 or n-6 PUFAs were expressed using relative percentages -that is, the distribution areas of $n-3$ or $n-6$ PUFAs peaks divided by the total peak areas of all detectable saturated and unsaturated free fatty acids (from the same sample) resolved from the gas chromatography column. Data are expressed as mean $\pm S D ; n=3$ per group. $\mathrm{AA}=$ arachidonic acid; $\mathrm{ALA}=\mathrm{a}-\mathrm{lipoic}$ acid; $\mathrm{DPA}=$ docosapentaenoic acid; $\mathrm{DHA}=$ docosahexaenoic acid; $E P A=e i c o s a p e n t a e n o i c$ acid; $L A=l i n o l e i c$ acid. $B$ The mapping data for this analysis was the centralized and standardized gene expression (FPKM), and the color gradient from blue to red indicates the change of gene expression from low to high. And make KEGG pathway term level2 annotations based on all differentially expressed genes. Horizontal: clustering between samples, reflecting the repeatability of samples. Longitudinal: Gene clustering, reflecting the similarity of different gene functions. Different square color blocks corresponded to specific gene enrichment pathways. 


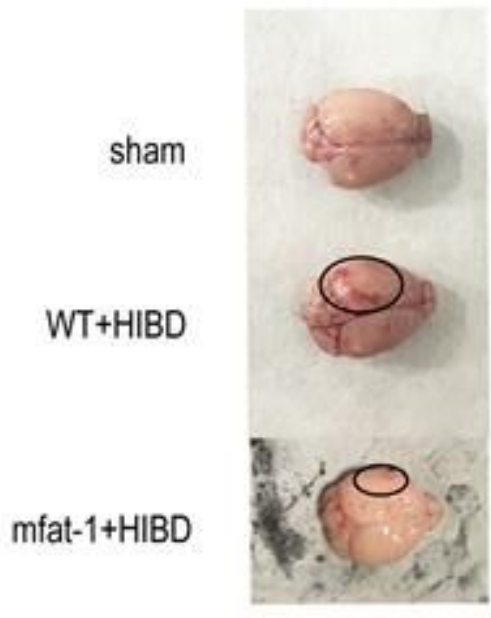

C
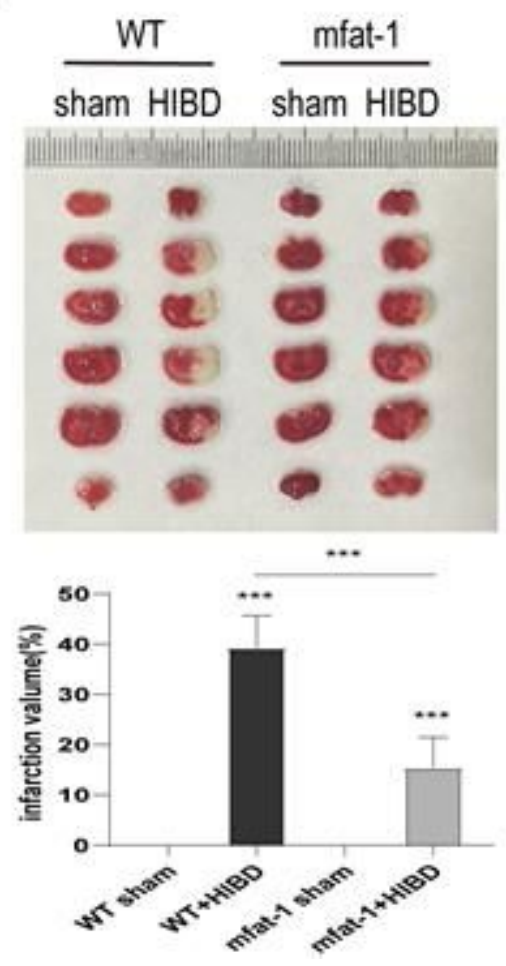

B

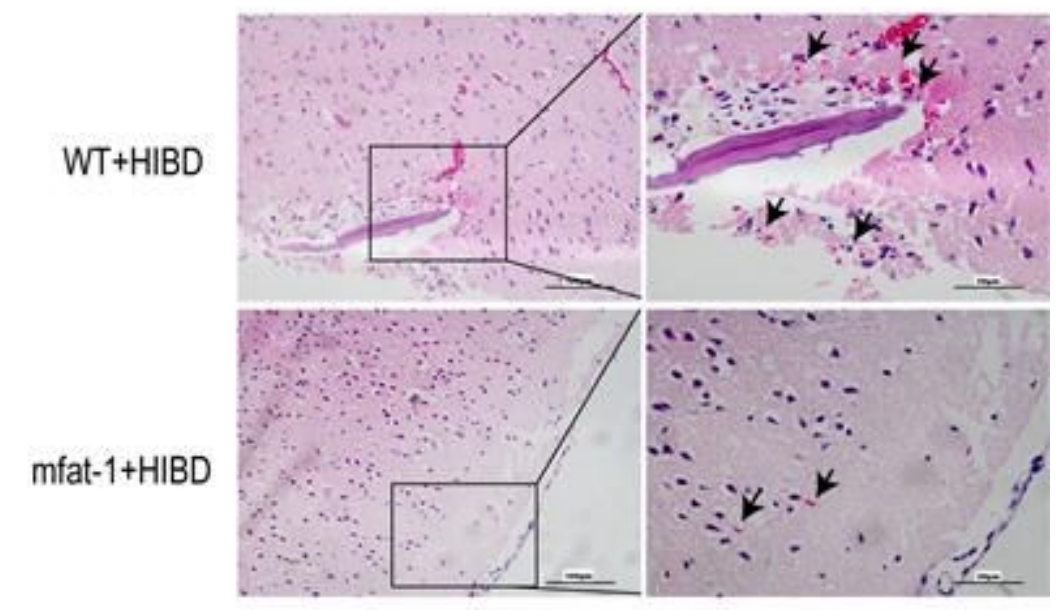

D

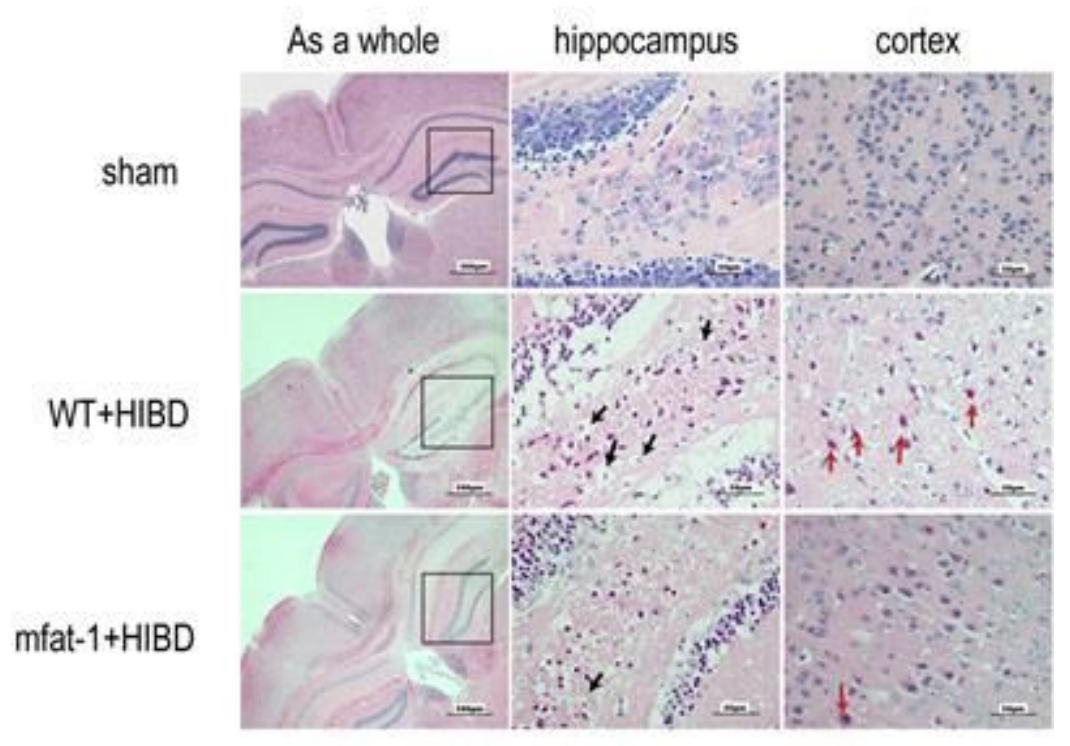

\section{Figure 2}

High ratio of n-3/n-6 in mfat- 1 transgenic mice showed neuroprotective effects after HIBD. A The picture showed that at 24 hours after HIBD, more severe diffuse hemorrhage and edema occurred in the WT group (Circled). B H/E staining showed cerebral cortex hemorrhage after HIBD. In the WT group, the edge of the cortex was damaged, the boundary was blurred, and there were scattered bleeding points (as shown by the arrow). Scale $=100 u m$ in left panel; Scale $=50 u m$ in the right panel. $C$ TTC staining in the coronal section of typical cerebral infarction at $24 \mathrm{~h}$ after HIBD. The infarct was seen in white and the normal tissue in red. Statistics in the bar chart below showed quantification of infarct volume in mfat-1 groups and WT groups. The data were expressed as the mean \pm SD; $n=5$ per group. $D H / E$ staining indicated neuronal morphology and pathological characteristics in ischemic penumbra at $24 \mathrm{~h}$ after 
HIBD. The red arrows represented typical red neurons with shrunken bodies, highly condensed chromatin, and vacuolated cytoplasm.

A

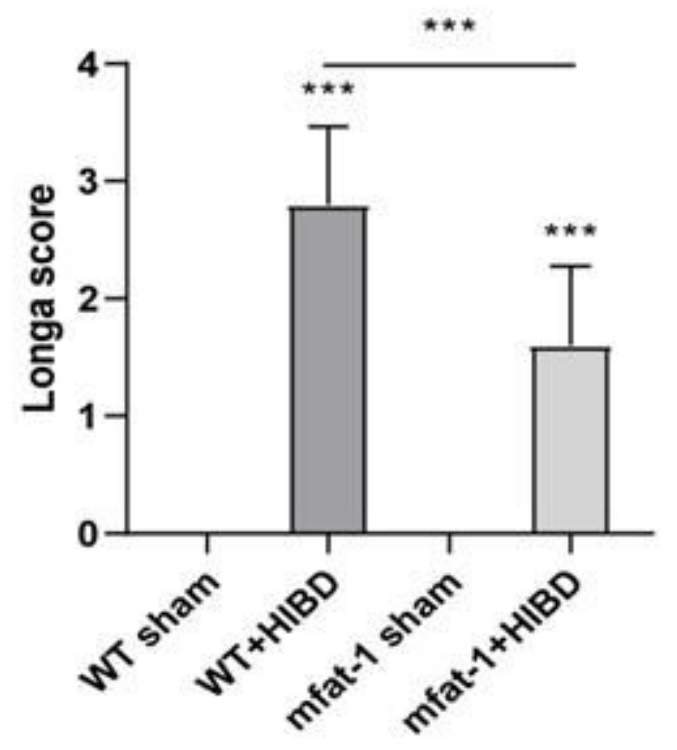

B

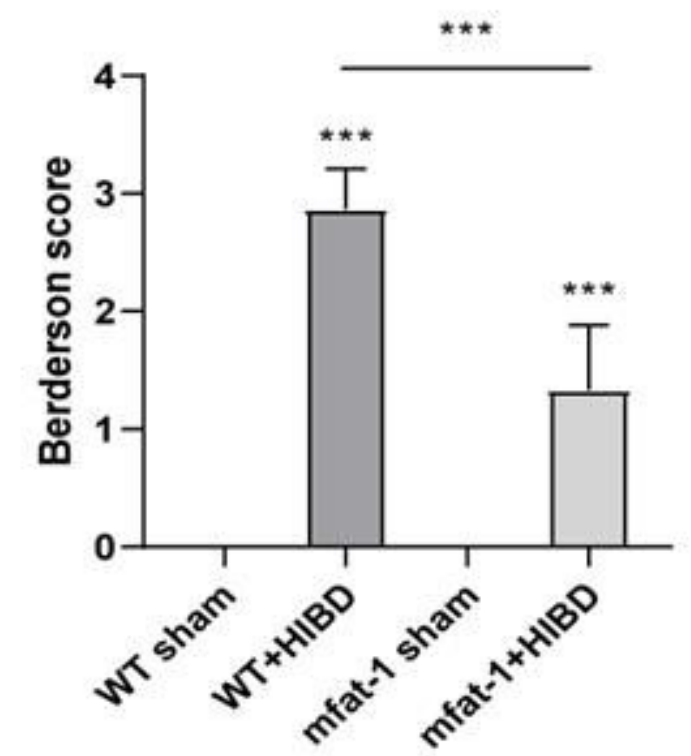

C

D
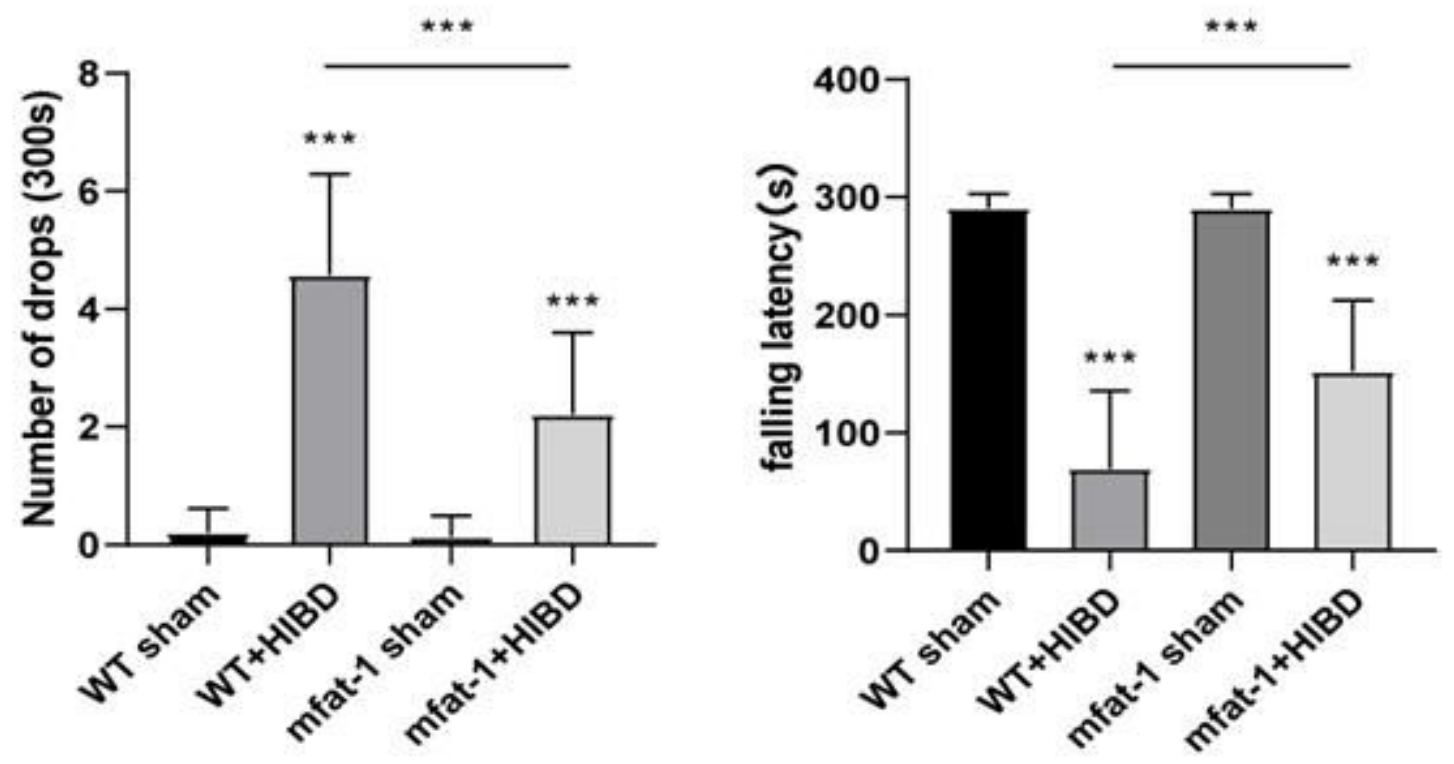

Figure 3

High ratio of n-3/n-6 PUFAs in mfat- 1 transgenic mice on HIBD improved neurobehavioral scores. A Longa 5-point scale B Bederson score; All values were expressed as mean scores $\pm S D ; n=30$ per group. C, D represented rotarod fallen times and fallen latency time within 300s; All values were expressed as mean $\pm S D ; n=15$ per group. 
A

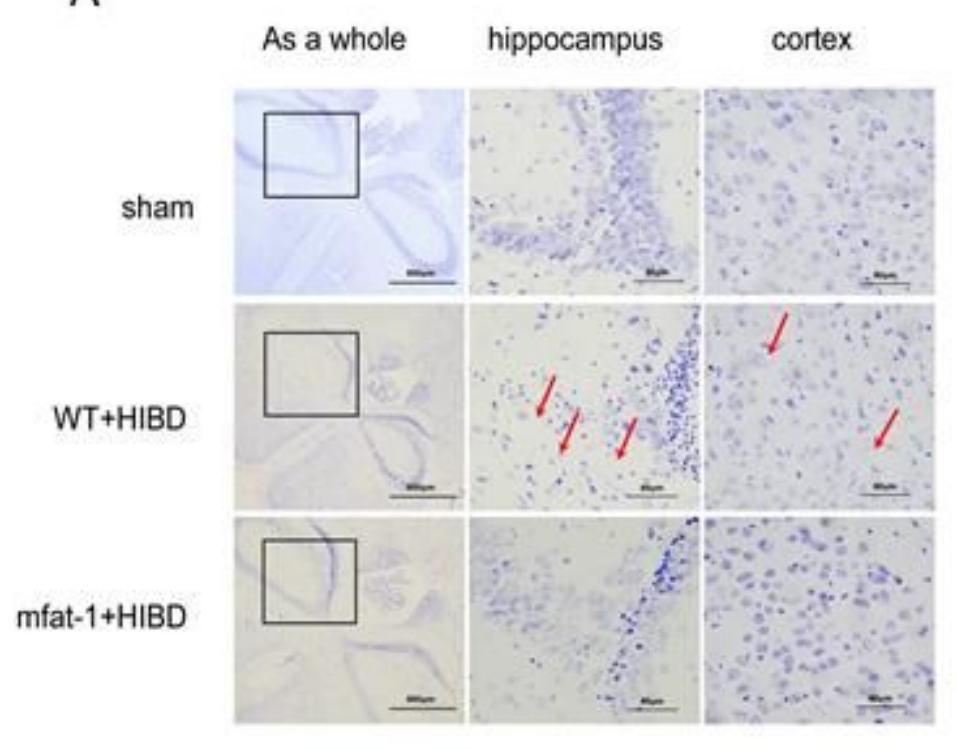

C

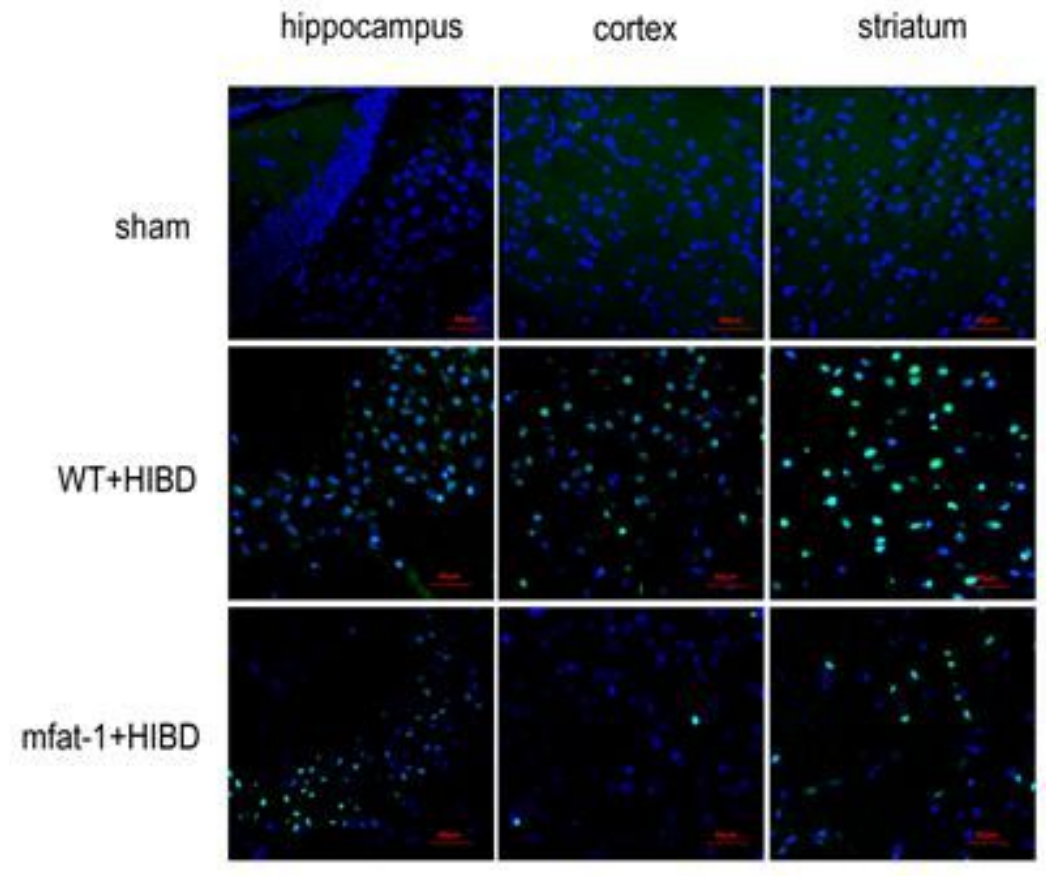

B
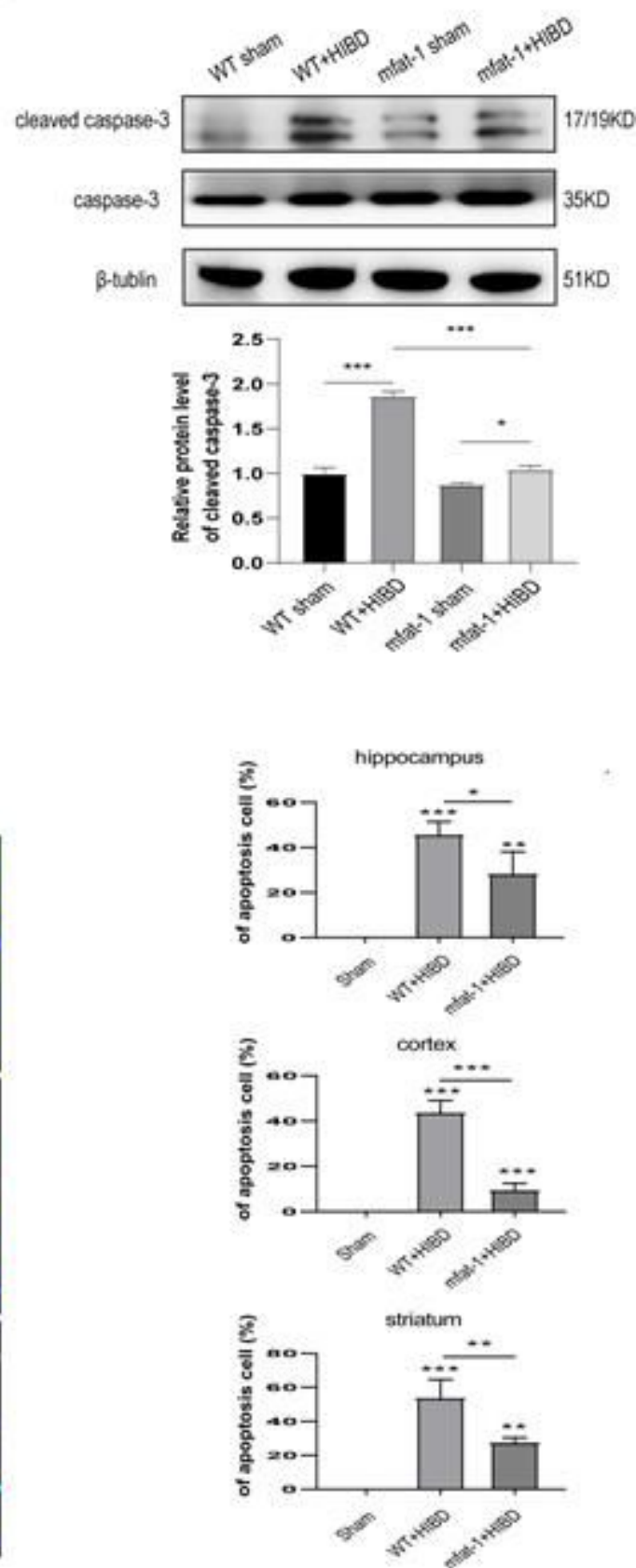

\section{Figure 4}

High ratio of n-3/n-6 PUFAs in mfat- 1 transgenic mice protected neurons against HIBD-induced neuronal apoptosis. A Nissl staining showed changes of morphological neurons in the ischemic penumbra of mice at $24 \mathrm{~h}$ after HIBD. The red arrow pointed to damaged neurons with shrunken bodies, concentrated nuclei, pale cytoplasm, and vacuoles. Scale $=500 \mathrm{um}$ in the left panel; Scale $=50 \mathrm{um}$ in the middle and right panels. B Western blotting analysis of cleaved caspase- 3 protein expression of the ischemic penumbra $24 \mathrm{~h}$ after reperfusion. The lower panel showed cleaved caspase- 3 and the corresponding $\beta$-tubulin bands (representative). The histogram in the panel below showed the results of the densitometric analysis. The data were expressed as the mean $\pm S D ; n=3$ per group. C. Left: representative photomicrographs showing TUNEL staining in the ischemic penumbra of after HIBD. Scale $=50 \mathrm{um}$. Right: the percentage of TUNEL- 
positive cells in the hippocampus, cortex and striatum respectively. The data were expressed as the mean $\pm \mathrm{SD} ; \mathrm{n}=5$ per group.

A

\section{TNF- $\alpha$}

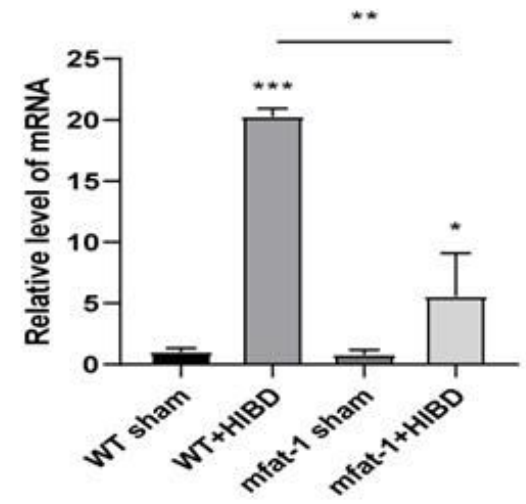

D

TNF- $\alpha$

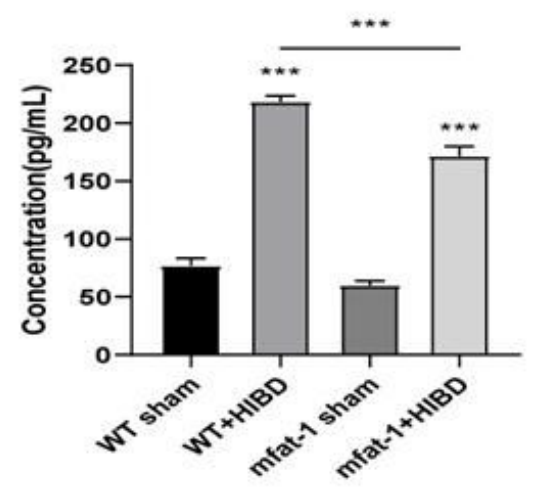

B

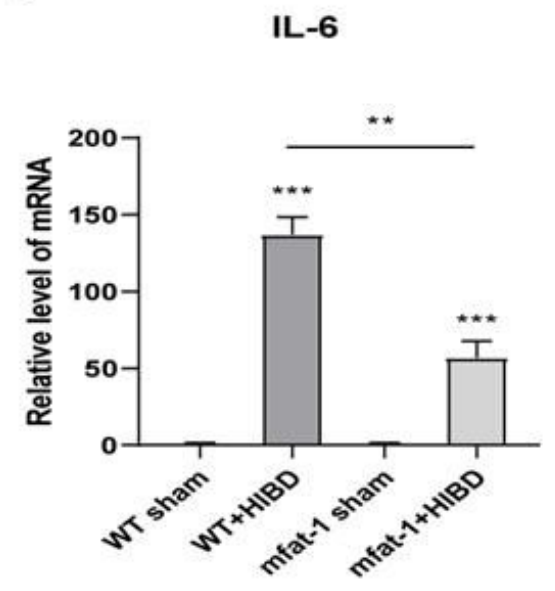

$\mathrm{E}$

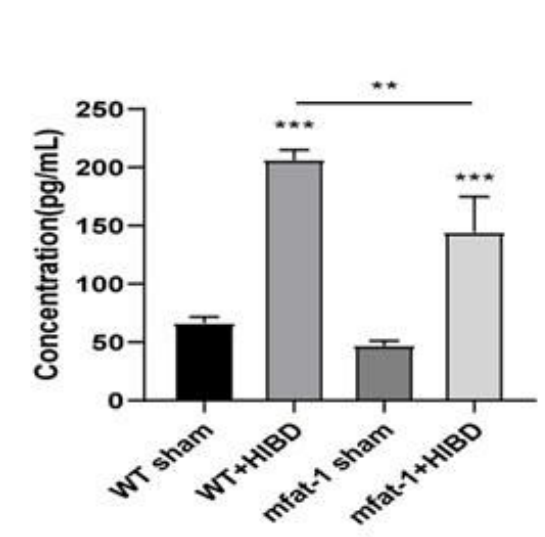

C

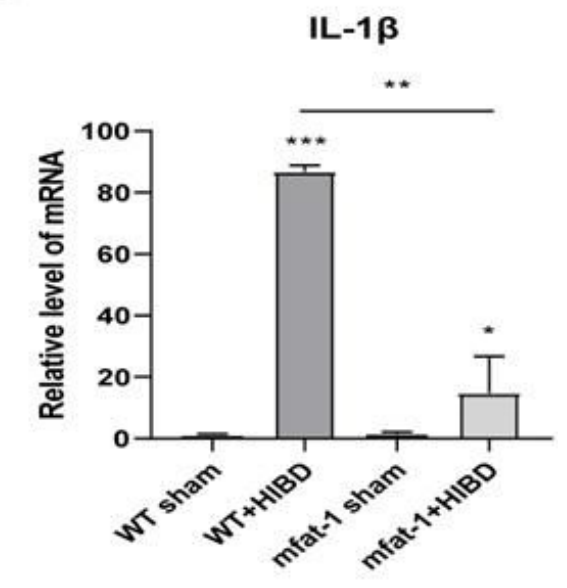

$\mathrm{F}$

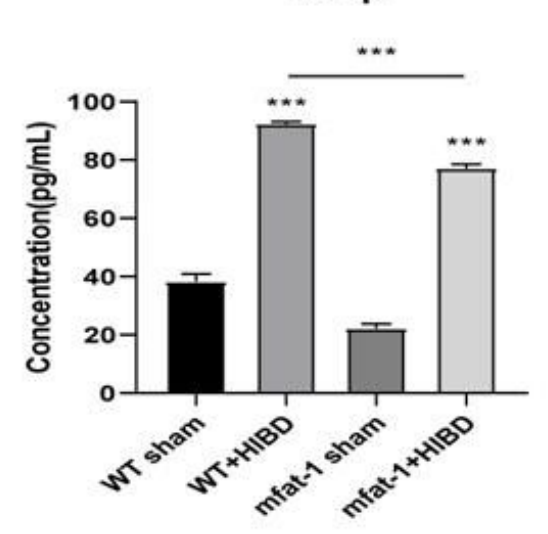

\section{Figure 5}

High ratio of n-3/n-6 PUFAs in mfat- 1 transgenic mice reduced inflammatory cytokine release after HIBD. $A, B, C$ Representation of the fold changes of the mRNA expression levels of IL-1 $\beta$, IL- 6 and TNF- $a$. mfat-1 group inhibited the upregulation of IL-1 $\beta$, IL- 6 and TNF-a compared to WT+HIBD groups. Average values represented the mean $\pm S D ; n=5$ per group. $D$, E F Expression levels of pro-inflammatory factors, IL- $1 \beta$, IL6 and TNF- $\alpha$ in groups were measured by ELISA assay. Data were presented as the means \pm SD; $n=5$ per group. 
A
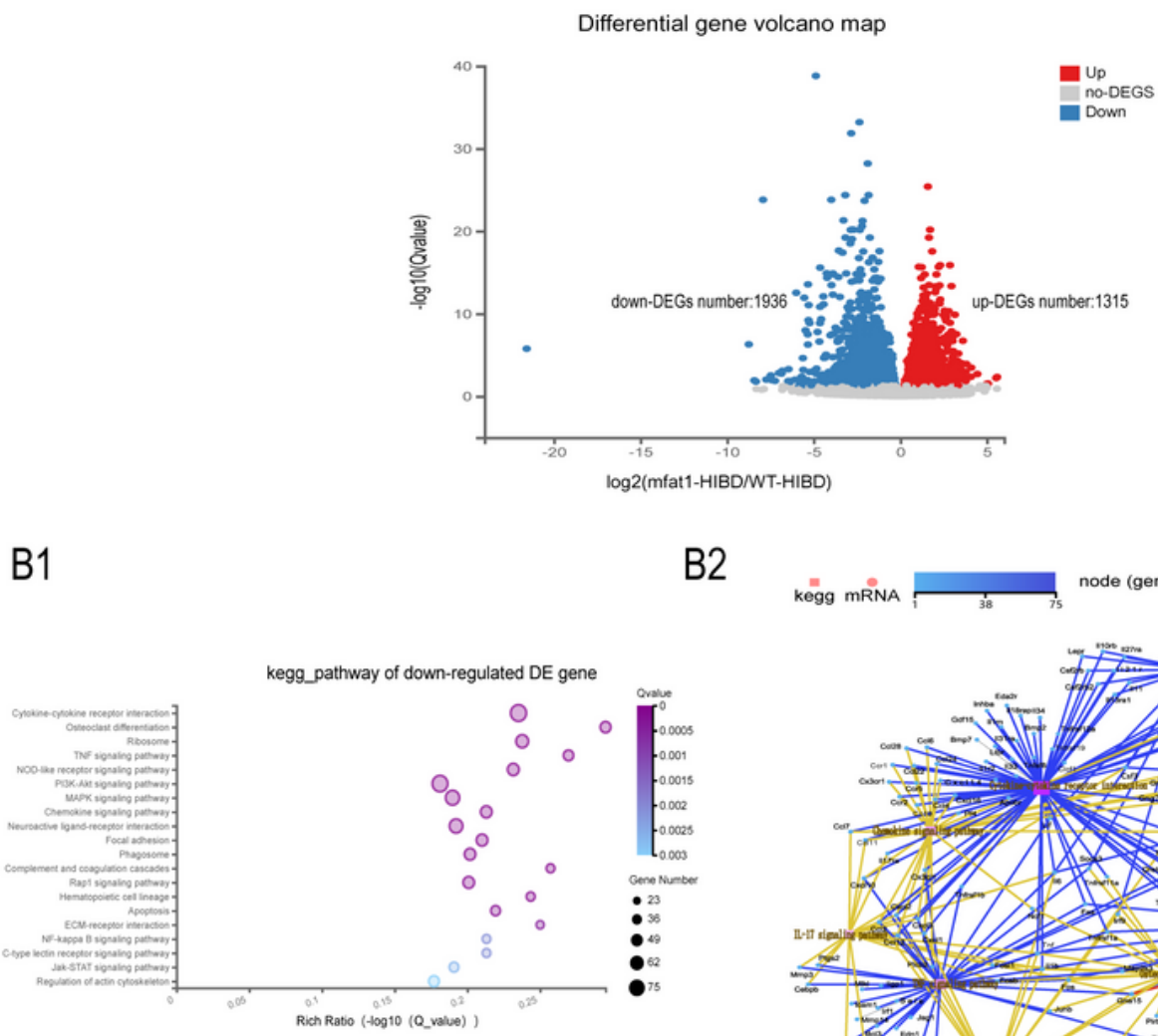

C1

B2
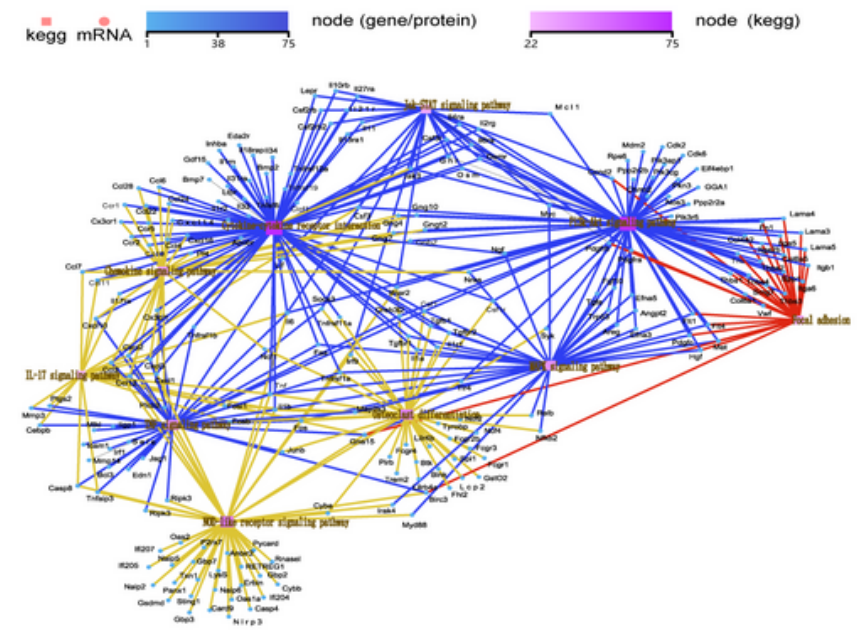

C2
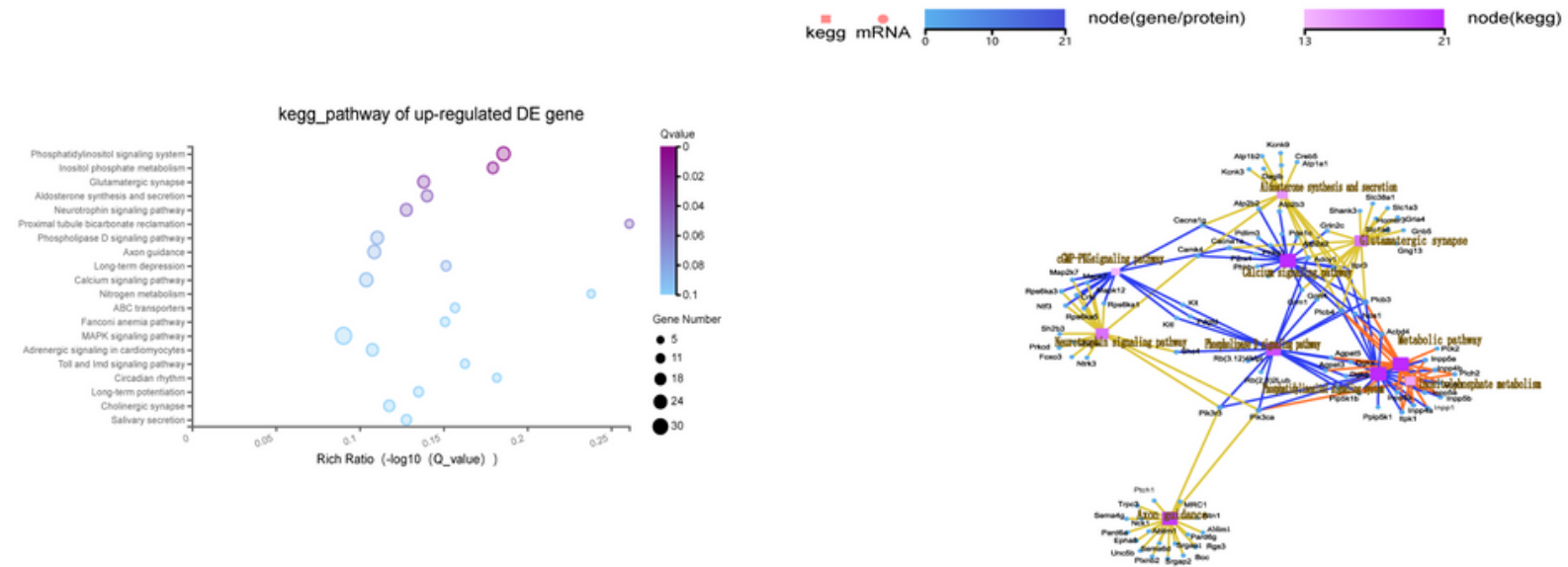

\section{Figure 6}

RNA sequencing analysis of DEGs. A Volcano map analysis of all DEGs in WT+HIBD and mfat- $1+H I B D$ groups; The horizontal axis was log2 fold change (mfat-1-HIBD/WT-HIBD), the vertical axis was -log10 ( $Q$ value), and each point represented a gene. Blue dots represented down-regulated genes; red dots represented up-regulated genes. B1 KEGG pathway analysis of differential down-regulated genes between WT+HIBD and mfat-1+HIBD groups; B2 Enrichment of KEGG pathways. The network was visualized using 
Cytoscape 3.7.1; C1 KEGG pathway analysis of differential up-regulated between WT+HIBD and mfat$1+\mathrm{HIBD} ; \mathrm{C} 2$ Enrichment of KEGG pathways. The network was visualized using Cytoscape 3.7.1. The phyper function in $\mathrm{R}$ software was used for enrichment analysis, $\mathrm{P}$ value was calculated, and $\mathrm{P}$ value was then FDR corrected. Generally, functions with $Q$ value $\leq 0.05$ ware regarded as significant enrichment.

A

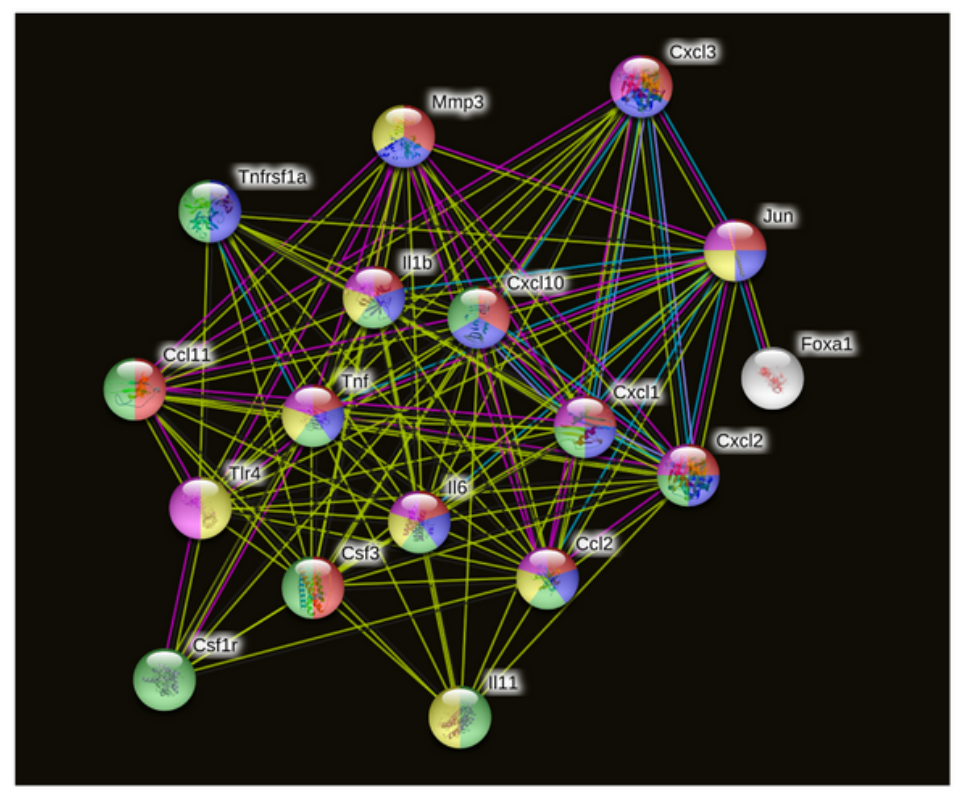

C

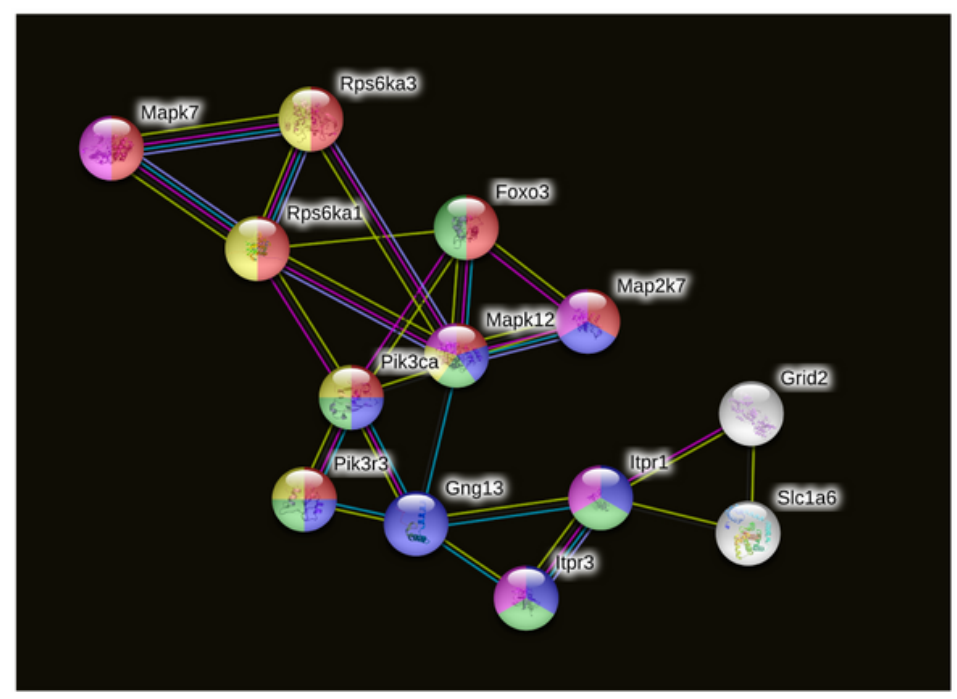

B

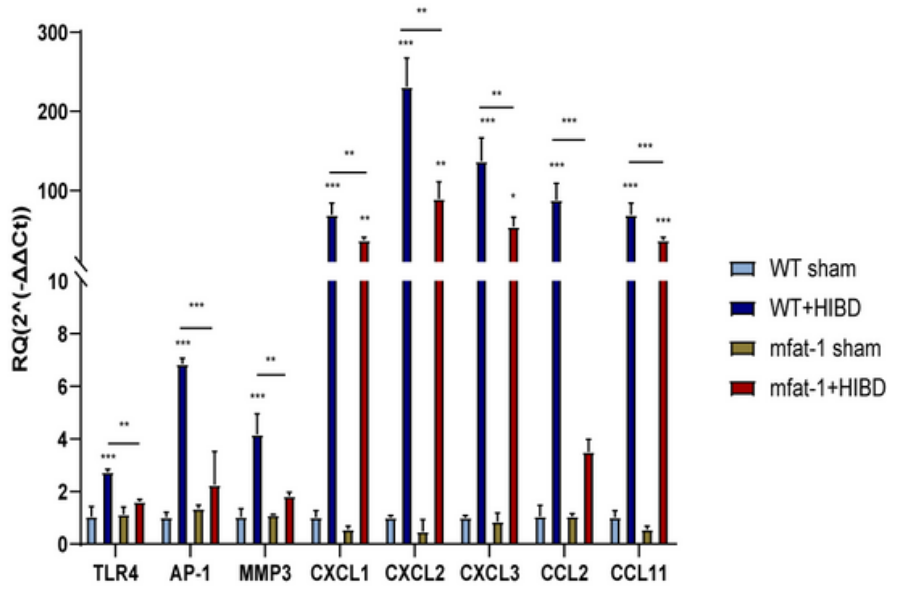

D

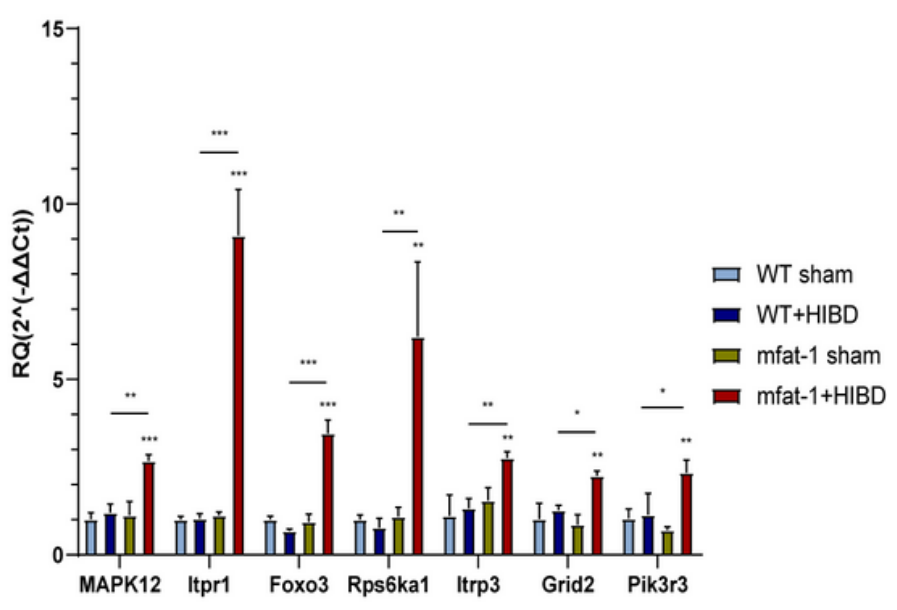

\section{Figure 7}

Verification of the DEGs. A Protein-protein interaction network among differential down-regulated genes obtained from RNA sequencing. Colors of the inside nodes indicate that the genes came from different significant KEGG pathways above. Edges represented protein-protein associations. B Transcriptional level expression of down-regulated differential genes by qPCR in groups $n=3$ per group. C Protein-protein interaction network among differential up-regulated genes obtained from RNA sequencing. Colors of the inside nodes indicated that the genes came from different significant KEGG pathways above. Edges 
represented protein-protein associations. D Transcriptional level expression of up-regulated differential genes by qPCR in groups, $n=3$ per group.

A

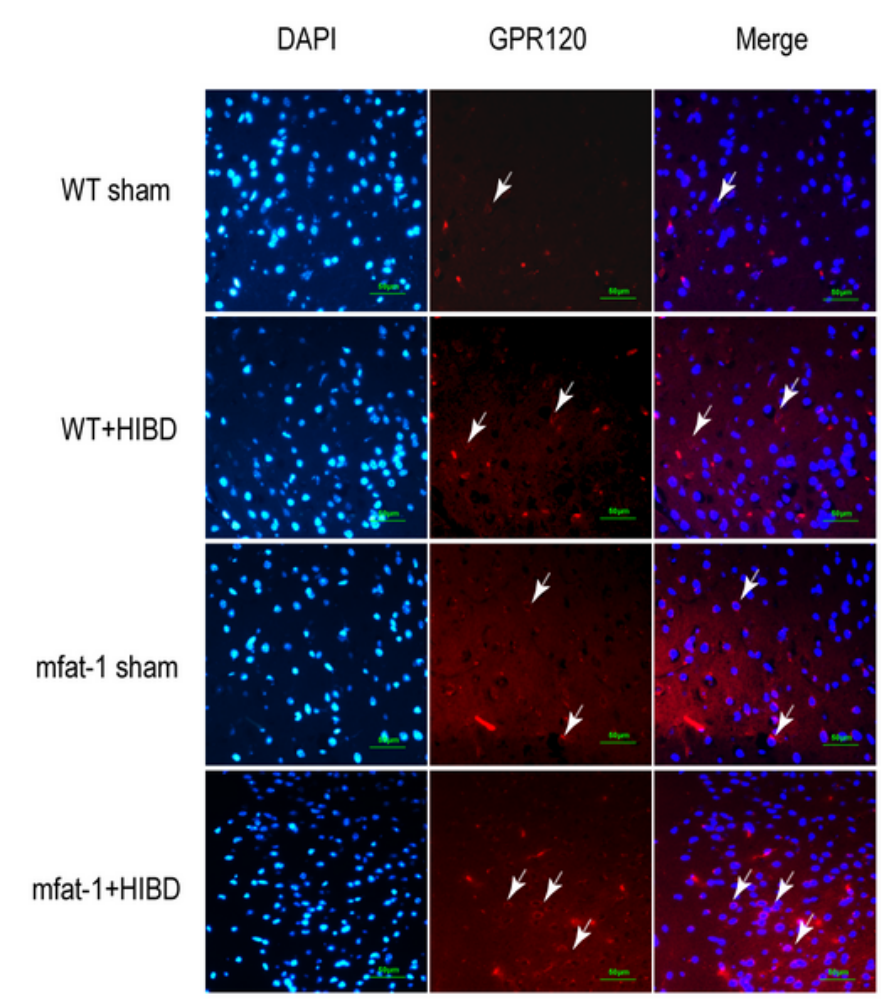

D

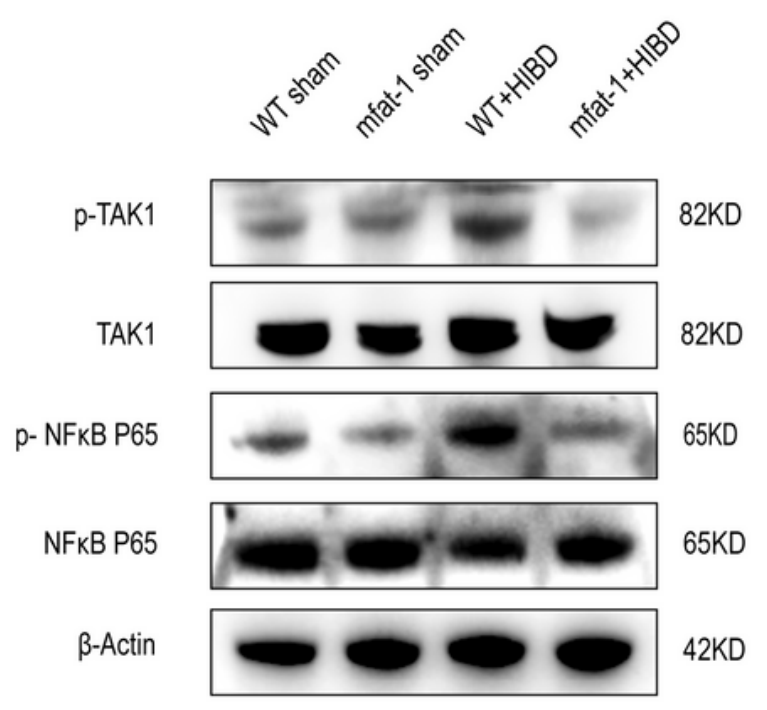

B

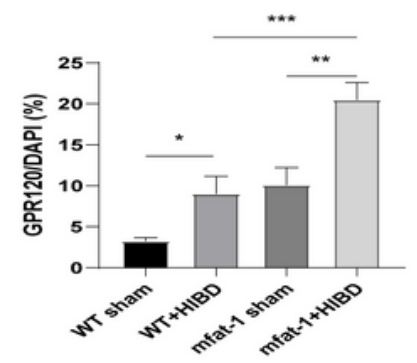

C
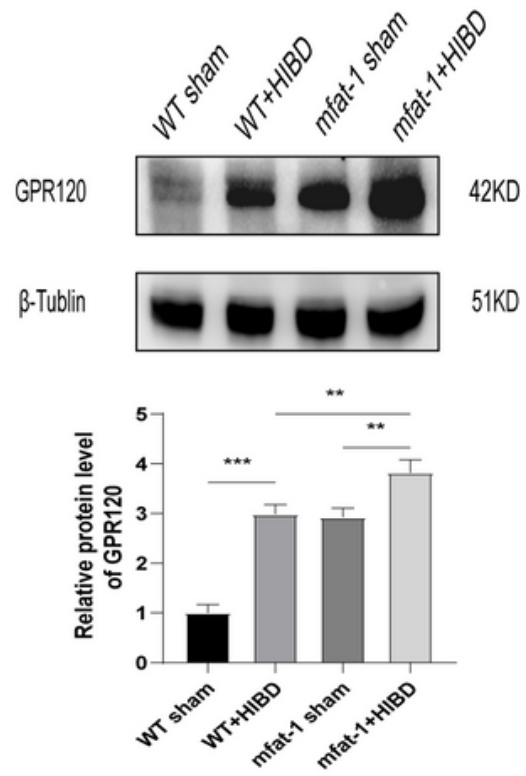

$\mathrm{E}$
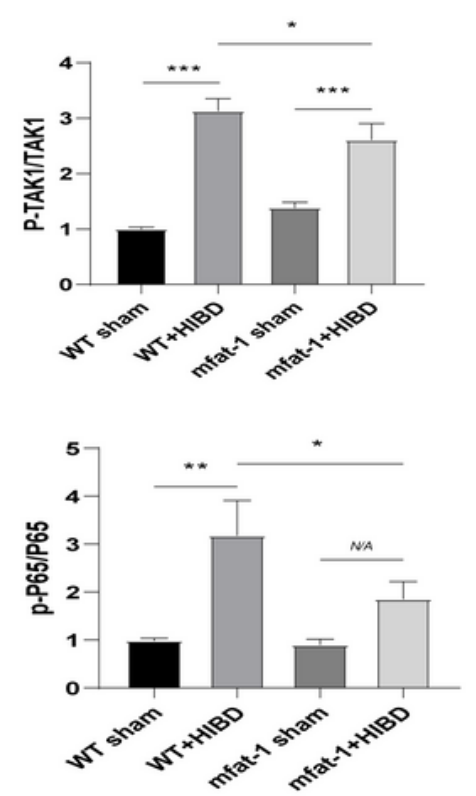

Figure 8

Activation of GPR120, suppression of TAK1 and NF-KB signaling protected against HIBD in mfat-1 mice by alleviating inflammation. A Immunofluorescence analysis was performed to study the expression level of GPR120 in the cerebral cortex (red). Nuclei were fluorescently labeled with DAPI (blue). Scale bar = 
50um. B Quantitative analysis of GPR120 protein immunofluorescence experiment. The results were expressed as the number of GPR120+ neurons/DAPI (\%); The data were expressed as the mean \pm SD $\llbracket n=$ 5 per group. $C$ Western blot analysis and quantification of GPR120 protein levels in brain tissues of different groups. The data were expressed as the mean $\pm S D ; n=3$ per group. D, E Western blot analysis and quantification of TAK1 and NF-KB P65 corresponding phosphorylation p-NF-KB P65 and p-TAK1 protein levels in brain tissues of different groups. The data were expressed as the mean $\pm S D ; n=3$ per group.

\section{Supplementary Files}

This is a list of supplementary files associated with this preprint. Click to download.

- figure8westernblotoriginalexposureimage.tif

- figure4westernblotoriginalexposureimage.tif

- 3.Additionalfiles.docx 